\title{
Growth performance of fourteen potato varieties as affected by arsenic contamination
}

\author{
Md. Nazmul Haque*, Md. Hazrat Ali, Tuhin Suvra Roy, Sheikh Muhammad Masum, \\ Md. Nousad Hossain
}

Department of Agronomy, Faculty of Agriculture, Sher-e-Bangla Agricultural University, Sher-e-Bangla Nagar, Dhaka-1207, Bangladesh

Email address:

sumon2539@gmail.com (M. N. Haque), hazratali11@yahoo.com (M. H. Ali), tuhinsuvra2002@yahoo.com (T. S. Roy), smmasum607@yahoo.com (S. M. Masum),nousad37@gmail.com (M. N. Hossain)

\section{To cite this article:}

Md. Nazmul Haque, Md. Hazrat Ali, Tuhin Suvra Roy, Sheikh Muhammad Masum, Md. Nousad Hossain. Growth Performance of Fourteen Potato Varieties as Affected by Arsenic Contamination. Journal of Plant Sciences. Vol. 3, No. 1, 2015, pp. 31-44.

doi: $10.11648 /$ j.jps.20150301.16

\begin{abstract}
The different levels of arsenic (As) had significant effect on growth contributing parameters of potato varieties. All parameters studied in this experiment were decreased with the increasing As levels. The results showed that though most of the growth parameters decreased with the increasing As levels but remained statistically similar up to $25 \mathrm{mg} \mathrm{As} \mathrm{kg}^{-1} \mathrm{soil}$ and thereafter drastically decreased. Among the potato varieties, Felsina gave the maximum number of stems hill ${ }^{-1}$, stem diameter, leaf area plant ${ }^{-1}$ and also chlorophyll content (SPAD value) of leaves, irrespective of As levels. Among the treatment

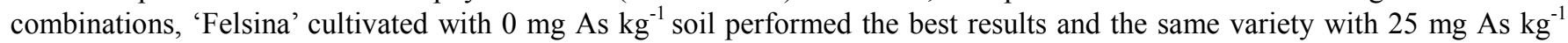
soil also showed the statistical similar results in terms of growth parameters.
\end{abstract}

Keywords: Arsenic contamination, Chlorophyll content, Phytotoxicity, Potato variety

\section{Introduction}

Arsenic (As) is a widespread natural element, which is not a bioorganic element to plants [1]. High As contamination of surface and groundwater occurs worldwide and has become a sociopolitical issue in several parts of the globe [2]. Among the countries facing As contamination problems, Bangladesh is the most affected [3]. Irrigation with As-enriched groundwater is therefore the main pathway for As to enter the human food chain $[4,5]$ and this has led to a number of studies on transfer of As through the water-soil-crop-food system. Recent research suggests that a number of crops and vegetable plant species are reported to accumulate significant amount of As [6-15]. Considering plants, Marin et al. [16] stated that, at higher concentration, arsenic was toxic to most plants. It interfered with metabolic processes and inhibited plant growth and development through arsenic induced phytotoxicity [17]. In case of vegetables, the highest As accumulation was observed in potato, arum, amaranth, radish, lady's finger, cauliflower, brinjal where as lower level of As accumulation was observed in beans, green chilli, tomato, bitter guard, lemon, turmeric etc. due to the Ascontaminated irrigation water [18]. As contents of vegetables varied; those exceeding the food hygiene concentration limit of $1.0 \mathrm{mg} \mathrm{kg} \mathrm{g}^{-1}$ as described by Abedin et al. [19] included kachu sak (Colocasia antiquorum) (0.09-3.99 $\mathrm{mg} \mathrm{kg}^{-1}$ ), potatoes (Solanum tuberosum) (0.07-1.36 mg kg-1) and kalmi sak (Ipomoea reptoms) $\left(0.1-1.53 \mathrm{mg} \mathrm{kg}^{-1}\right)$. As is generally considered phytotoxic and is expected to negatively affect plant growth [20]. It was reported that As concentrations in agricultural plants varied from 0.007 to about $7.50 \mathrm{mg} \mathrm{kg}^{-1}$ $[7,11,21]$.

Potato (Solanum tuberosum L.) is the $4^{\text {th }}$ world crop after wheat, rice and maize. Bangladesh is the $7^{\text {th }}$ potato production country in the world [22]. The statistics available for the As contamination in ground water indicate that 59 districts (around $85 \%$ of the total area of Bangladesh) and about 75 million people are at risk [23]. People of As affected areas are consuming contaminated potatoes and creating serious problem of health. Several research have been examined the sources and behavior of As in different plants, but toxicity in potatoes and its impact on sustainable potato production are not established. Under this circumstance, the proposed study was undertaken to observe the effect of As on potato production. 


\section{Materials and Methods}

A pot experiment was conducted during the period from November 10, 2012 to February 18, 2013 in Rabi season. The experimental area was situated at $23^{0} 77^{\prime} \mathrm{N}$ latitude and $90^{\circ} 33^{\prime} \mathrm{E}$ longitude at an altitude of $8.6 \mathrm{~m}$ above the sea level. Fourteen potato varieties viz., Diamant $\left(\mathrm{V}_{1}\right)$, Cardinal $\left(\mathrm{V}_{2}\right)$, Asterix $\left(\mathrm{V}_{3}\right)$, Granola $\left(\mathrm{V}_{4}\right)$, Lady Rosetta $\left(\mathrm{V}_{5}\right)$, Courage $\left(\mathrm{V}_{6}\right)$, BARI TPS-1 $\left(\mathrm{V}_{7}\right)$, Meridian $\left(\mathrm{V}_{8}\right)$, Felsina $\left(V_{9}\right)$, Laura $\left(V_{10}\right)$, Quincy $\left(V_{11}\right)$, Sagitta $\left(V_{12}\right)$, Rumana $\left(\mathrm{V}_{13}\right)$, Jam Alu $\left(\mathrm{V}_{14}\right)$ and three arsenic levels namely $0 \mathrm{mg} \mathrm{As} \mathrm{kg}^{-1}$ soil $\left(\mathrm{As}_{0}\right), 25 \mathrm{mg} \mathrm{As} \mathrm{kg}{ }^{-1}$ soil $\left(\mathrm{As}_{1}\right), 50 \mathrm{mg} \mathrm{As} \mathrm{kg}{ }^{-1}$ soil $\left(\mathrm{As}_{2}\right)$ were selected for this experiment. The experiment was laid out in a Randomized Complete Block Design (RCBD) with three replications thus comprised 126 baskets. The size of each basket was $25 \mathrm{~cm}$ in diameter and $30 \mathrm{~cm}$ in height. For the Arsenic treatment in soil, sodium meta arsenate $\left(\mathrm{Na}_{2} \mathrm{HAsO}_{4} \cdot 7 \mathrm{H}_{2} \mathrm{O}\right)$ was used as the source of As. Leaf area plant ${ }^{-1}$ was measured by nondestructive method using CL-202 Leaf Area Meter (USA). Three mature plant of each pot were measured by using portable Chlorophyll Meter (SPAD-502, Minolta, Japan) and then calculated an average SPAD value for each pot at each sampling time. The data obtained for different characters were statistically analyzed following the analysis of variance (ANOVA) techniques by using MSTAT-C computer package programme. The significant differences among the treatment means were compared by Duncan's Multiple Range Test (DMRT) at 5\% level of probability [24].

\section{Results and Discussion}

\subsection{Days to Emergence (Visual Observation)}

Days to emergence was significantly influenced by the different potato varieties and/or As levels (Table 1 and 2). Table 1 exhibited that the variety 'Jam Alu' took the maximum duration (17.78 days) for emergence whereas, the minimum (10.22 days) was taken by 'Quincy'. This result showed that 'Quincy' was the early emergence variety whereas, 'Jam Alu' was the late one. It is may be due to varietal characters. On the other hand, duration of emergence increased with increasing As levels but $\mathrm{As}_{0}$ and $\mathrm{As}_{1}$ exhibited similar result. Similar trend of result was also found by Talukdar [25], who observed that the mean value of germination percentage, germination index and relative germination rate of Trigonella foenum-graecum L. and Lathyrus sativus L. decreased with concomitant increase of As levels. When plants were exposed to excess As either in soil or in solution culture, they exhibit toxicity symptoms such as inhibition of seed germination [17]. In case of treatment combinations the minimum duration for emergence (8.67 days) was recorded from the combination of 'Quincy' and $\mathrm{As}_{0}$ treatment which was statistically similar (9.33, 9.67 and 10.33 days, respectively) with $\mathrm{V}_{11} \mathrm{As}_{1}, \mathrm{~V}_{8} \mathrm{As}_{0}, \mathrm{~V}_{8} \mathrm{As}_{1}$ whereas, the maximum duration (21.00 days) was recorded from the combination of 'Jam Alu' and $50 \mathrm{mg} \mathrm{As} \mathrm{kg}^{-1}$ soil which was statistically similar (19.67 days) with $\mathrm{V}_{2} \mathrm{As}_{2}$ (Table 2).

Table 1. Effect of varieties and As levels on days to emergence and plant height of potato.

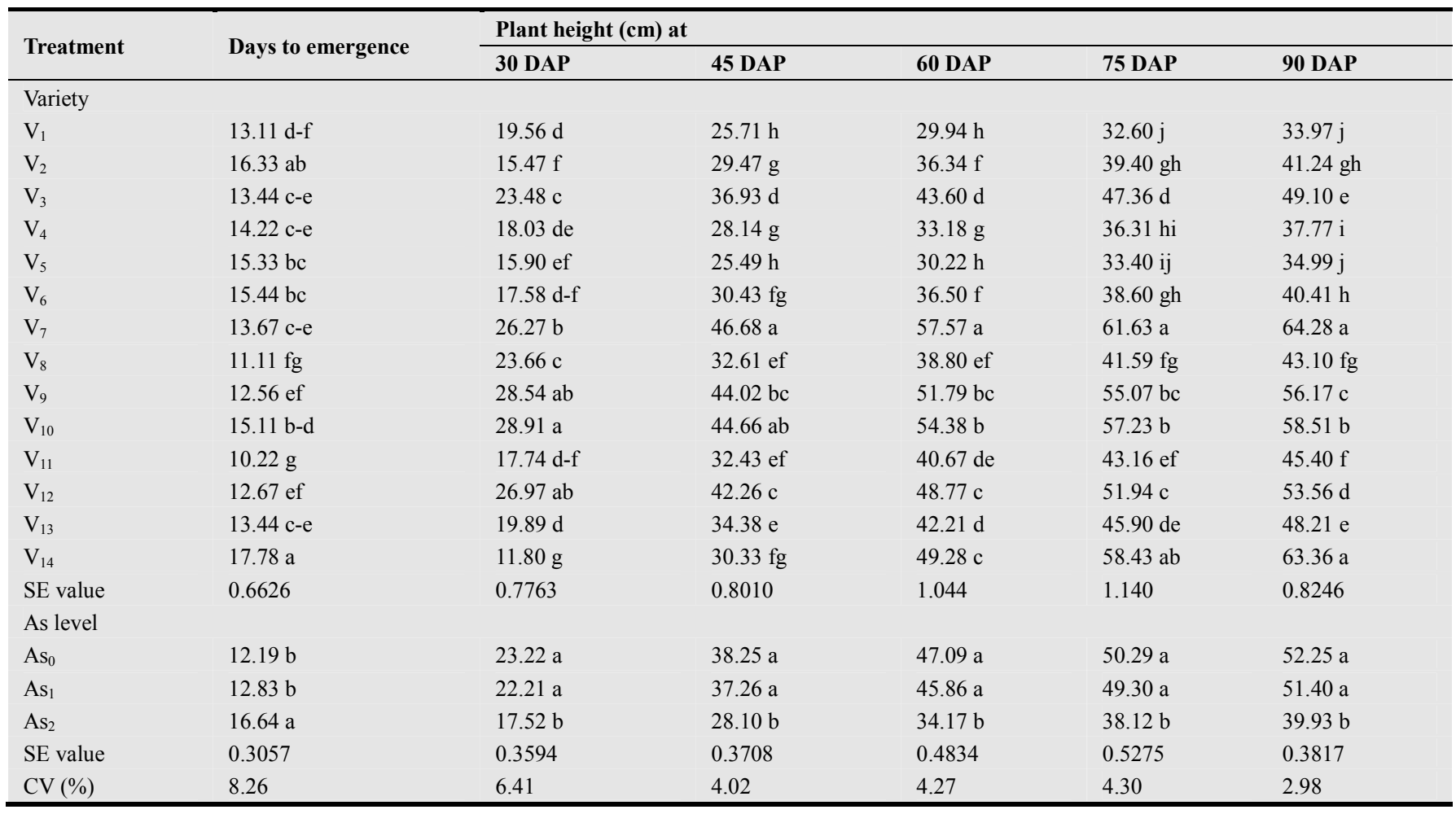

In a column means having similar letter (s) are statistically similar and those having dissimilar letter (s) differ significantly by DMRT at 0.05 level of probability 
Table 2. Interaction effect of varieties and As levels on days to emergence and plant height of potato.

\begin{tabular}{|c|c|c|c|c|c|c|}
\hline \multirow{2}{*}{ Variety $\times$ As level } & \multirow{2}{*}{ Days to emergence } & \multicolumn{5}{|c|}{ Plant height $(\mathrm{cm})$ at } \\
\hline & & 30 DAP & 45 DAP & 60 DAP & 75 DAP & 90 DAP \\
\hline $\mathrm{V}_{1} \mathrm{As}_{0}$ & $11.67 \mathrm{~m}-\mathrm{p}$ & $23.80 \mathrm{i}-\mathrm{k}$ & $29.60 \mathrm{mn}$ & 33.80 rs & $36.47 \mathrm{tu}$ & $37.93 \mathrm{uv}$ \\
\hline $\mathrm{V}_{1} \mathrm{As}_{1}$ & $12.001-\mathrm{o}$ & $22.87 \mathrm{j}-1$ & 28.87 no & $32.70 \mathrm{r}-\mathrm{t}$ & $35.73 \mathrm{t}-\mathrm{v}$ & $37.20 \mathrm{v}$ \\
\hline $\mathrm{V}_{1} \mathrm{As}_{2}$ & $15.67 \mathrm{f}-\mathrm{i}$ & $12.00 \mathrm{rs}$ & $18.67 \mathrm{~s}$ & $23.33 \mathrm{w}$ & $25.60 \mathrm{x}$ & $26.77 \mathrm{y}$ \\
\hline $\mathrm{V}_{2} \mathrm{As}_{0}$ & $14.33 \mathrm{~g}-\mathrm{k}$ & $18.10 \mathrm{n}-\mathrm{p}$ & $33.50 \mathrm{~g}-\mathrm{j}$ & $41.50 \mathrm{~m}-\mathrm{o}$ & 44.33 m-p & $45.50 \mathrm{o}-\mathrm{q}$ \\
\hline $\mathrm{V}_{2} \mathrm{As}_{1}$ & $15.00 \mathrm{f}-\mathrm{j}$ & $17.27 \mathrm{p}$ & $32.27 \mathrm{i}-1$ & 40.53 no & $43.23 n-q$ & $44.43 \mathrm{pq}$ \\
\hline $\mathrm{V}_{2} \mathrm{As}_{2}$ & $19.67 \mathrm{ab}$ & $11.03 \mathrm{st}$ & $22.63 \mathrm{r}$ & $27.00 \mathrm{v}$ & $30.63 \mathrm{w}$ & $33.80 \mathrm{w}$ \\
\hline $\mathrm{V}_{3} \mathrm{As}_{0}$ & $12.001-\mathrm{o}$ & $25.43 \mathrm{f}-\mathrm{i}$ & $41.63 \mathrm{~d}$ & $48.80 \mathrm{hi}$ & $52.03 \mathrm{f}-\mathrm{i}$ & $53.60 \mathrm{~g}-\mathrm{i}$ \\
\hline $\mathrm{V}_{3} \mathrm{As}_{1}$ & $12.33 \mathrm{k}-\mathrm{o}$ & $24.30 \mathrm{~h}-\mathrm{j}$ & $40.53 \mathrm{~d}$ & $47.80 \mathrm{~h}-\mathrm{j}$ & 51.17 g-j & 52.90 g-j \\
\hline $\mathrm{V}_{3} \mathrm{As}_{2}$ & $16.00 \mathrm{e}-\mathrm{h}$ & $20.701-n$ & $28.63 n-p$ & $34.20 \mathrm{q}-\mathrm{s}$ & $38.87 \mathrm{r}-\mathrm{t}$ & $40.80 \mathrm{st}$ \\
\hline $\mathrm{V}_{4} \mathrm{As}_{0}$ & $12.67 \mathrm{k}-\mathrm{n}$ & $20.03 \mathrm{~m}-\mathrm{o}$ & $31.77 \mathrm{j}-\mathrm{m}$ & $37.27 \mathrm{pq}$ & $40.20 \mathrm{q}-\mathrm{s}$ & 41.77 rs \\
\hline $\mathrm{V}_{4} \mathrm{As}_{1}$ & $13.33 \mathrm{i}-\mathrm{m}$ & 19.47 m-p & $30.87 \mathrm{k}-\mathrm{n}$ & $35.87 \mathrm{qr}$ & $39.00 \mathrm{r}-\mathrm{t}$ & $40.73 \mathrm{st}$ \\
\hline $\mathrm{V}_{4} \mathrm{As}_{2}$ & $16.67 \mathrm{c}-\mathrm{f}$ & $14.60 \mathrm{q}$ & $21.80 \mathrm{r}$ & $26.40 \mathrm{v}$ & $29.73 \mathrm{w}$ & $30.80 x$ \\
\hline $\mathrm{V}_{5} \mathrm{As}_{0}$ & $13.33 \mathrm{j}-\mathrm{m}$ & $18.50 \mathrm{n}-\mathrm{p}$ & $29.801-n$ & $34.67 \mathrm{qr}$ & $38.17 \mathrm{~s}-\mathrm{u}$ & $39.67 \mathrm{~s}-\mathrm{v}$ \\
\hline $\mathrm{V}_{5} \mathrm{As}_{1}$ & $14.00 \mathrm{~h}-1$ & 17.53 op & 28.90 no & 33.80 rs & $37.17 \mathrm{~s}-\mathrm{u}$ & $38.67 \mathrm{t}-\mathrm{v}$ \\
\hline $\mathrm{V}_{5} \mathrm{As}_{2}$ & $18.67 \mathrm{bc}$ & $11.67 \mathrm{rs}$ & $17.77 \mathrm{~s}$ & $22.20 \mathrm{w}$ & $24.87 x$ & $26.63 \mathrm{y}$ \\
\hline $\mathrm{V}_{6} \mathrm{As}_{0}$ & 13.67 i-m & 19.40 m-p & $34.77 \mathrm{f}-\mathrm{i}$ & $41.30 \mathrm{~m}-\mathrm{o}$ & $43.10 \mathrm{o}-\mathrm{q}$ & $44.60 \mathrm{pq}$ \\
\hline $\mathrm{V}_{6} \mathrm{As}_{1}$ & $14.33 \mathrm{~g}-\mathrm{k}$ & 18.57 n-p & 33.87 g-j & 39.90 op & 41.90 p-r & $44.00 \mathrm{qr}$ \\
\hline $\mathrm{V}_{6} \mathrm{As}_{2}$ & $18.33 \mathrm{~b}-\mathrm{d}$ & $14.77 \mathrm{q}$ & $22.67 \mathrm{r}$ & $28.30 \mathrm{uv}$ & $30.80 \mathrm{w}$ & $32.63 \mathrm{wx}$ \\
\hline $\mathrm{V}_{7} \mathrm{As}_{0}$ & $12.001-\mathrm{o}$ & $28.07 \mathrm{~b}-\mathrm{e}$ & $49.60 \mathrm{a}$ & $62.57 \mathrm{a}$ & $66.03 \mathrm{a}$ & $69.43 \mathrm{a}$ \\
\hline $\mathrm{V}_{7} \mathrm{As}_{1}$ & $12.67 \mathrm{k}-\mathrm{n}$ & $27.03 \mathrm{~d}-\mathrm{g}$ & $48.60 \mathrm{a}$ & $60.93 \mathrm{ab}$ & $65.07 \mathrm{ab}$ & $68.77 \mathrm{ab}$ \\
\hline $\mathrm{V}_{7} \mathrm{As}_{2}$ & $16.33 \mathrm{~d}-\mathrm{g}$ & $23.70 \mathrm{i}-\mathrm{k}$ & $41.83 \mathrm{~d}$ & $49.20 \mathrm{~g}-\mathrm{i}$ & $53.80 \mathrm{e}-\mathrm{g}$ & $54.63 \mathrm{f}-\mathrm{h}$ \\
\hline $\mathrm{V}_{8} \mathrm{As}_{0}$ & $9.67 \mathrm{pr}$ & $25.80 \mathrm{e}-\mathrm{i}$ & $36.80 \mathrm{ef}$ & $43.60 \mathrm{k}-\mathrm{n}$ & $46.47 \mathrm{k}-\mathrm{o}$ & $47.73 \mathrm{~m}-\mathrm{o}$ \\
\hline $\mathrm{V}_{8} \mathrm{As}_{1}$ & 10.33 o-r & $24.63 \mathrm{~g}-\mathrm{j}$ & $35.23 \mathrm{e}-\mathrm{h}$ & $42.471-\mathrm{o}$ & $45.571-\mathrm{o}$ & 46.90 n-p \\
\hline $\mathrm{V}_{8} \mathrm{As}_{2}$ & $13.33 \mathrm{j}-\mathrm{m}$ & $20.531-n$ & $25.80 \mathrm{q}$ & 30.33 tu & $32.73 \mathrm{vw}$ & $34.67 \mathrm{w}$ \\
\hline $\mathrm{V}_{9} \mathrm{As}_{0}$ & $11.00 \mathrm{n}-\mathrm{q}$ & $31.00 \mathrm{a}$ & $48.27 \mathrm{ab}$ & $57.73 \mathrm{~cd}$ & $59.83 \mathrm{c}$ & $60.73 \mathrm{~cd}$ \\
\hline $\mathrm{V}_{9} \mathrm{As}_{1}$ & $11.67 \mathrm{~m}-\mathrm{p}$ & 30.57 a & $47.07 \mathrm{a}-\mathrm{c}$ & $55.80 \mathrm{de}$ & $58.80 \mathrm{~cd}$ & $59.87 \mathrm{~d}$ \\
\hline $\mathrm{V}_{9} \mathrm{As}_{2}$ & $15.00 \mathrm{f}-\mathrm{j}$ & $24.07 \mathrm{~h}-\mathrm{k}$ & 36.73 ef & $41.831-\mathrm{o}$ & $46.57 \mathrm{k}-\mathrm{o}$ & $47.90 \mathrm{~m}-\mathrm{o}$ \\
\hline $\mathrm{V}_{10} \mathrm{As}_{0}$ & $13.33 \mathrm{j}-\mathrm{m}$ & $30.43 \mathrm{ab}$ & $48.53 \mathrm{a}$ & $60.00 \mathrm{a}-\mathrm{c}$ & $61.83 \mathrm{c}$ & $63.00 \mathrm{c}$ \\
\hline $\mathrm{V}_{10} \mathrm{As}_{1}$ & $14.00 \mathrm{~h}-1$ & $29.73 \mathrm{a}-\mathrm{c}$ & $47.70 \mathrm{ab}$ & $58.80 \mathrm{~b}-\mathrm{d}$ & $61.10 \mathrm{c}$ & $62.07 \mathrm{~cd}$ \\
\hline $\mathrm{V}_{10} \mathrm{As}_{2}$ & $18.00 \mathrm{~b}-\mathrm{e}$ & $26.57 \mathrm{e}-\mathrm{h}$ & $37.73 \mathrm{e}$ & $44.33 \mathrm{k}-\mathrm{m}$ & $48.77 \mathrm{i}-1$ & $50.47 \mathrm{j}-1$ \\
\hline $\mathrm{V}_{11} \mathrm{As}_{0}$ & $8.67 \mathrm{r}$ & $19.80 \mathrm{~m}-\mathrm{p}$ & 35.70 e-g & $46.00 \mathrm{i}-\mathrm{k}$ & 47.93 j-m & $49.63 \mathrm{k}-\mathrm{m}$ \\
\hline $\mathrm{V}_{11} \mathrm{As}_{1}$ & $9.33 \mathrm{qr}$ & 18.87 n-p & $34.90 \mathrm{f}-\mathrm{h}$ & $44.97 \mathrm{j}-1$ & $46.83 \mathrm{k}-\mathrm{n}$ & $48.771-n$ \\
\hline $\mathrm{V}_{11} \mathrm{As}_{2}$ & $12.67 \mathrm{k}-\mathrm{n}$ & $14.57 \mathrm{q}$ & $26.70 \mathrm{o}-\mathrm{q}$ & $31.03 \mathrm{~s}-\mathrm{u}$ & $34.70 \mathrm{uv}$ & $37.80 \mathrm{v}$ \\
\hline $\mathrm{V}_{12} \mathrm{As}_{0}$ & $11.00 \mathrm{n}-\mathrm{q}$ & $29.00 \mathrm{a}-\mathrm{d}$ & $46.00 \mathrm{bc}$ & 53.67 ef & $56.00 \mathrm{de}$ & $57.50 \mathrm{e}$ \\
\hline $\mathrm{V}_{12} \mathrm{As}_{1}$ & $11.67 \mathrm{~m}-\mathrm{p}$ & $27.40 \mathrm{c}-\mathrm{f}$ & $45.13 \mathrm{c}$ & $52.63 \mathrm{f}$ & 54.97 ef & 56.87 ef \\
\hline $\mathrm{V}_{12} \mathrm{As}_{2}$ & $15.33 \mathrm{f}-\mathrm{j}$ & $24.50 \mathrm{~h}-\mathrm{j}$ & $35.63 \mathrm{e}-\mathrm{g}$ & 40.00 op & $44.87 \mathrm{~m}-\mathrm{p}$ & $46.30 \mathrm{n}-\mathrm{q}$ \\
\hline $\mathrm{V}_{13} \mathrm{As}_{0}$ & $11.67 \mathrm{~m}-\mathrm{p}$ & $21.77 \mathrm{k}-\mathrm{m}$ & 36.80 ef & $46.27 \mathrm{i}-\mathrm{k}$ & $49.97 \mathrm{~h}-\mathrm{k}$ & $52.63 \mathrm{~h}-\mathrm{j}$ \\
\hline $\mathrm{V}_{13} \mathrm{As}_{1}$ & $12.33 \mathrm{k}-\mathrm{o}$ & $20.33 \mathrm{mn}$ & $35.80 \mathrm{e}-\mathrm{g}$ & $45.00 \mathrm{j}-1$ & $48.93 \mathrm{i}-1$ & $51.57 \mathrm{i}-\mathrm{k}$ \\
\hline $\mathrm{V}_{13} \mathrm{As}_{2}$ & $16.33 \mathrm{~d}-\mathrm{g}$ & 17.57 op & $30.53 \mathrm{k}-\mathrm{n}$ & $35.37 \mathrm{qr}$ & $38.80 \mathrm{r}-\mathrm{t}$ & $40.43 \mathrm{~s}-\mathrm{u}$ \\
\hline $\mathrm{V}_{14} \mathrm{As}_{0}$ & $15.67 \mathrm{f}-\mathrm{i}$ & $13.97 \mathrm{qr}$ & 32.80 h-k & $52.03 \mathrm{fg}$ & $61.73 \mathrm{bc}$ & $67.80 \mathrm{ab}$ \\
\hline $\mathrm{V}_{14} \mathrm{As}_{1}$ & $16.67 c-f$ & $12.37 \mathrm{q}-\mathrm{s}$ & $31.87 \mathrm{j}-\mathrm{m}$ & $50.90 \mathrm{f}-\mathrm{h}$ & $60.67 \mathrm{c}$ & $66.87 \mathrm{~b}$ \\
\hline $\mathrm{V}_{14} \mathrm{As}_{2}$ & $21.00 \mathrm{a}$ & $9.07 \mathrm{t}$ & $26.33 \mathrm{pq}$ & $44.90 \mathrm{j}-1$ & $52.90 \mathrm{e}-\mathrm{h}$ & $55.40 \mathrm{e}-\mathrm{g}$ \\
\hline SE value & 0.6603 & 0.7763 & 0.8010 & 1.044 & 1.140 & 0.8246 \\
\hline CV (\%) & 8.26 & 6.41 & 4.02 & 4.27 & 4.30 & 2.98 \\
\hline
\end{tabular}

In a column means having similar letter (s) are statistically similar and those having dissimilar letter (s) differ significantly by DMRT at 0.05 level of probability 


\subsection{Plant Height}

The plant height of potato was significantly influenced by varieties and/or As levels at 15, 30, 45, 60, 75 and 90 (at harvest) DAP (Table 1 and 2). It was observed that plant height increased with advancing growing period irrespective of varieties. Plant height increased rapidly at the early stages of growth; however, rate of progression in height was slow at the later stages except 'Jam Alu'. The variations in the plant height among the varieties also recorded by Rabbani [26] and Bashar [27] in their experimental results. On the other hand, plant height decreased with increasing As levels but $\mathrm{As}_{0}$ and $\mathrm{As}_{1}$ statistically similar results. The phytotoxicity at lower soil As concentrations was not significant. Stimulation of growth by As additions has been reported to increase growth of potatoes [28]. It is possible that displacement of soil phosphate by arsenate increased the availability of phosphate to the plant, which results in the increase of plant growth [28, 29]. Thus, Kabata-Pendias and Pendias [30] recommended

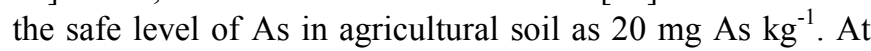
higher concentration, As is toxic to most plants. It interferes with metabolic processes and inhibits plant growth and development through As induced phytotoxicity [16]. When plants are exposed to excess As either in soil or in solution culture, they exhibit toxicity symptoms such as decrease in plant height [31-34]. In case of treatment combinations Table 2 exhibited that, the highest plant height $(69.43 \mathrm{~cm})$ was observed from the 'BARI TPS-1' with $\mathrm{As}_{0}$ treatment combination which was statistically similar $(68.77 \mathrm{~cm}$ and $67.80 \mathrm{~cm}$, respectively) with $\mathrm{V}_{7} \mathrm{As}_{1}$ and $\mathrm{V}_{14} \mathrm{As}_{0}$ whereas, the lowest plant $(26.63 \mathrm{~cm})$ was obtained from the 'Lady

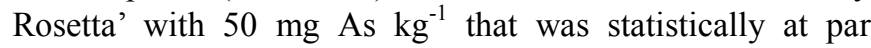
$(26.77 \mathrm{~cm})$ with $\mathrm{V}_{1} \mathrm{As}_{2}$ at harvesting stage. Such type of discontinuity in increasing plant height might have been caused by the variation between varieties in response to As or might be due to differences in crop growth pattern. The study disclosed that 'BARI TPS-1' variety treated with $\mathrm{As}_{0}$ performed the best result in terms of plant height.

\subsection{Number of Leaves Plant ${ }^{1}$}

Different varieties and/or As levels exhibited significant variation in respect to number of leaves plant ${ }^{-1}$ at $30,45,60$, 75 and 90 DAP (Figure 1, 2 and Table 3). It is observed from figure 1 that number of leaves plant ${ }^{-1}$ increased with advancing growing period up to 75 DAP irrespective of varieties and thereafter decreased due to senescence of leaves. The study referred that 'Jam Alu' produced maximum number of leaf. On the other hand, from figure 2 it is also found that number of leaves plant ${ }^{-1}$ increased with advancing growing period up to 75 DAP irrespective of As levels and thereafter decreased due to senescence of leaves. Present study showed that number of leaves plant ${ }^{-1}$ was not statistically affected up to $25 \mathrm{mg} \mathrm{As} \mathrm{kg}^{-1}$ soil treatment compared to control but at higher concentration (50 mg As $\mathrm{kg}^{-1}$ soil) leaves number significantly decreased. In case of treatment combinations (Table 3), number of leaves plant ${ }^{-1}$ increased with advancing growing period upto 75 DAP irrespective of varieties and As levels and thereafter decreased. At harvest, the maximum leaves number plant $^{-1}$ (167.3) was obtained from the 'Jam Alu' with $\mathrm{As}_{0}$ combination treatment which was statistically similar (166.3) with $\mathrm{V}_{14} \mathrm{As}_{1}$ whereas, the minimum (26.67) was recorded from the combination of 'Courage' with $50 \mathrm{mg} \mathrm{As} \mathrm{kg}^{-1}$ soil which was statistically at par (30.33) with $\mathrm{V}_{4} \mathrm{As}_{2}$.

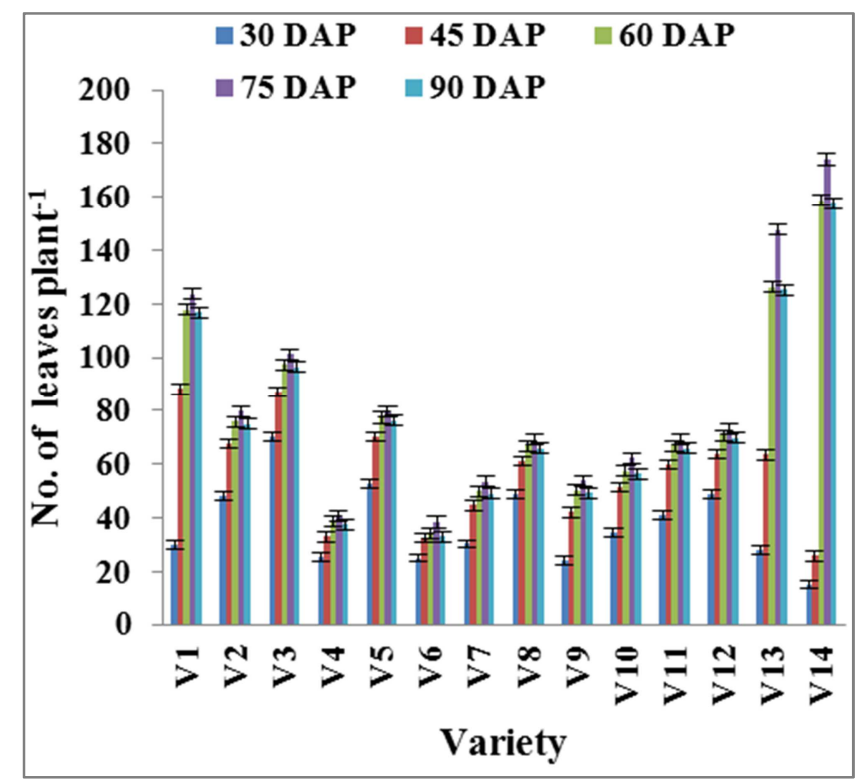

Figure 1. Effect of varieties on number of leaves plant ${ }^{-1}$ at different growth stages of potato (Vertical bar represents $S E$ value).

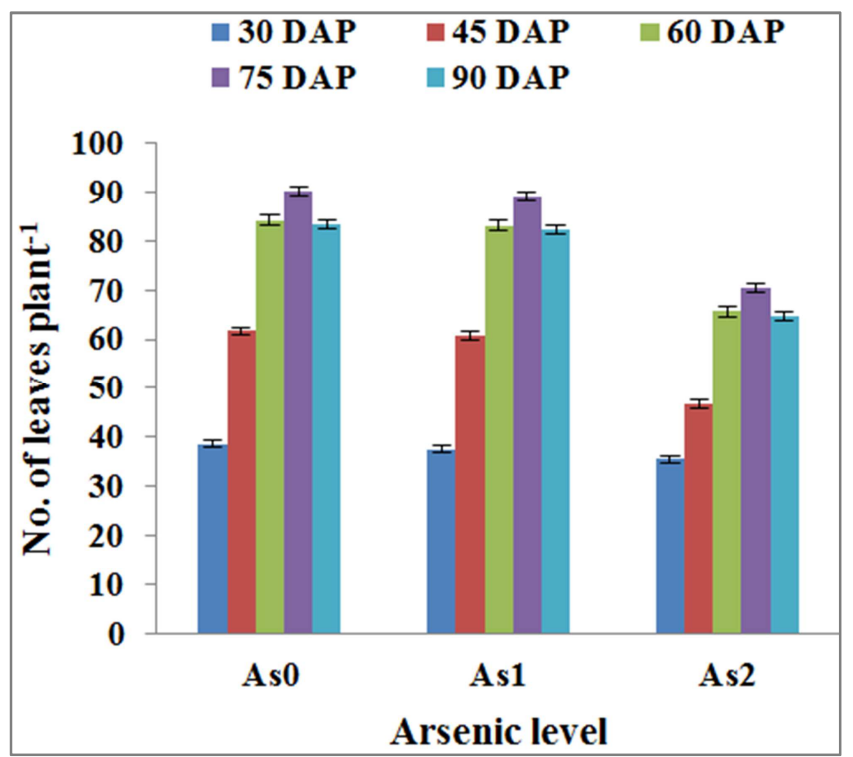

Figure 2. Effect of As levels on number of leaves plant ${ }^{1}$ at different growth stages of potato (Vertical bar represents $S E$ value). 
Table 3. Interaction effect of varieties and As levels on number of leaves plant ${ }^{1}$ at different DAP of potato.

\begin{tabular}{|c|c|c|c|c|c|}
\hline \multirow{2}{*}{ Variety $\times$ As level } & \multicolumn{5}{|c|}{ Number of leaves plant ${ }^{-1}$ at } \\
\hline & 30 DAP & 45 DAP & 60 DAP & 75 DAP & 90 DAP \\
\hline $\mathrm{V}_{1} \mathrm{As}_{0}$ & $31.33 \mathrm{~h}-1$ & 96.33 a & $125.7 \mathrm{~d}$ & $130.7 \mathrm{~d}$ & $124.7 \mathrm{~d}$ \\
\hline $\mathrm{V}_{1} \mathrm{As}_{1}$ & $30.33 \mathrm{i}-\mathrm{n}$ & $95.33 \mathrm{ab}$ & $123.7 \mathrm{~d}$ & $129.7 \mathrm{~d}$ & $122.7 \mathrm{~d}$ \\
\hline $\mathrm{V}_{1} \mathrm{As}_{2}$ & $28.33 \mathrm{j}-\mathrm{q}$ & $72.67 \mathrm{~d}-\mathrm{g}$ & $104.0 \mathrm{f}$ & $110.7 \mathrm{e}$ & $103.0 \mathrm{f}$ \\
\hline $\mathrm{V}_{2} \mathrm{As}_{0}$ & $49.33 \mathrm{~b}-\mathrm{d}$ & $73.33 \mathrm{~d}-\mathrm{f}$ & $82.67 \mathrm{gh}$ & $85.67 \mathrm{fg}$ & $81.67 \mathrm{gh}$ \\
\hline $\mathrm{V}_{2} \mathrm{As}_{1}$ & $48.33 \mathrm{~cd}$ & $72.33 \mathrm{~d}-\mathrm{g}$ & $81.67 \mathrm{~g}-\mathrm{i}$ & $84.67 \mathrm{f}-\mathrm{h}$ & $80.67 \mathrm{~g}-\mathrm{i}$ \\
\hline $\mathrm{V}_{2} \mathrm{As}_{2}$ & $46.33 \mathrm{de}$ & $57.00 \mathrm{j}$ & 63.331 & $68.67 \mathrm{jk}$ & 62.331 \\
\hline $\mathrm{V}_{3} \mathrm{As}_{0}$ & $71.67 \mathrm{a}$ & $91.33 \mathrm{ab}$ & $103.7 \mathrm{f}$ & $107.3 \mathrm{e}$ & $102.7 \mathrm{f}$ \\
\hline $\mathrm{V}_{3} \mathrm{As}_{1}$ & 70.67 a & $90.33 \mathrm{~b}$ & $102.7 \mathrm{f}$ & $106.3 \mathrm{e}$ & $101.7 \mathrm{f}$ \\
\hline $\mathrm{V}_{3} \mathrm{As}_{2}$ & $68.33 \mathrm{a}$ & $79.33 \mathrm{c}$ & $86.00 \mathrm{~g}$ & $89.67 \mathrm{f}$ & $85.00 \mathrm{~g}$ \\
\hline $\mathrm{V}_{4} \mathrm{As}_{0}$ & 26.67 k-r & $36.00 \mathrm{p}$ & $42.67 n-p$ & $44.67 n-p$ & $41.67 \mathrm{n}-\mathrm{p}$ \\
\hline $\mathrm{V}_{4} \mathrm{As}_{1}$ & 25.67 m-r & $35.00 \mathrm{pq}$ & $41.67 \mathrm{n}-\mathrm{p}$ & $43.67 \mathrm{o}-\mathrm{q}$ & $40.67 n-p$ \\
\hline $\mathrm{V}_{4} \mathrm{As}_{2}$ & 23.67 p-r & $27.67 \mathrm{~s}$ & $31.33 \mathrm{qr}$ & $33.00 \mathrm{~s}$ & $30.33 \mathrm{qr}$ \\
\hline $\mathrm{V}_{5} \mathrm{As}_{0}$ & $54.00 \mathrm{~b}$ & $76.67 \mathrm{~cd}$ & $85.33 \mathrm{~g}$ & $87.33 \mathrm{f}$ & $84.33 \mathrm{~g}$ \\
\hline $\mathrm{V}_{5} \mathrm{As}_{1}$ & $53.00 \mathrm{bc}$ & $75.67 \mathrm{c}-\mathrm{e}$ & $84.33 \mathrm{~g}$ & $86.33 \mathrm{f}$ & $83.33 \mathrm{~g}$ \\
\hline $\mathrm{V}_{5} \mathrm{As}_{2}$ & $50.33 \mathrm{~b}-\mathrm{d}$ & $58.00 \mathrm{j}$ & 62.331 & $64.67 \mathrm{kl}$ & 61.331 \\
\hline $\mathrm{V}_{6} \mathrm{As}_{0}$ & $26.331-\mathrm{r}$ & $34.67 \mathrm{pq}$ & 37.67 op & $41.00 \mathrm{pq}$ & $36.67 \mathrm{op}$ \\
\hline $\mathrm{V}_{6} \mathrm{As}_{1}$ & 25.33 n-r & 33.67 p-r & $36.67 \mathrm{pq}$ & 40.00 p-r & $35.67 \mathrm{pq}$ \\
\hline $\mathrm{V}_{6} \mathrm{As}_{2}$ & $23.33 \mathrm{qr}$ & $29.00 \mathrm{rs}$ & $27.67 \mathrm{r}$ & 34.33 rs & $26.67 \mathrm{r}$ \\
\hline $\mathrm{V}_{7} \mathrm{As}_{0}$ & $31.67 \mathrm{~h}-\mathrm{k}$ & $50.67 \mathrm{k}-\mathrm{m}$ & $57.00 \mathrm{~lm}$ & $61.67 \mathrm{~lm}$ & $56.00 \mathrm{~lm}$ \\
\hline $\mathrm{V}_{7} \mathrm{As}_{1}$ & 30.67 i-m & $49.331-n$ & $56.00 \mathrm{~m}$ & $60.67 \mathrm{~lm}$ & $55.00 \mathrm{~m}$ \\
\hline $\mathrm{V}_{7} \mathrm{As}_{2}$ & $28.67 \mathrm{j}-\mathrm{p}$ & 33.67 p-r & $37.00 \mathrm{o}-\mathrm{q}$ & $38.33 \mathrm{q}-\mathrm{s}$ & $36.00 \mathrm{o}-\mathrm{q}$ \\
\hline $\mathrm{V}_{8} \mathrm{As}_{0}$ & $50.33 \mathrm{~b}-\mathrm{d}$ & 64.67 hi & $71.00 \mathrm{jk}$ & $73.33 \mathrm{ij}$ & $70.00 \mathrm{jk}$ \\
\hline $\mathrm{V}_{8} \mathrm{As}_{1}$ & $49.00 \mathrm{~b}-\mathrm{d}$ & $63.67 \mathrm{i}$ & $70.00 \mathrm{k}$ & $72.33 \mathrm{j}$ & $69.00 \mathrm{k}$ \\
\hline $\mathrm{V}_{8} \mathrm{As}_{2}$ & $47.00 \mathrm{de}$ & $54.67 \mathrm{j}-1$ & $59.33 \mathrm{~lm}$ & $61.67 \mathrm{~lm}$ & $58.33 \mathrm{~lm}$ \\
\hline $\mathrm{V}_{9} \mathrm{As}_{0}$ & 25.00 o-r & $46.00 \mathrm{~m}-\mathrm{o}$ & $54.33 \mathrm{~m}$ & $57.33 \mathrm{~m}$ & $53.33 \mathrm{~m}$ \\
\hline $\mathrm{V}_{9} \mathrm{As}_{1}$ & 24.00 p-r & 45.00 no & $53.33 \mathrm{~m}$ & $56.33 \mathrm{~m}$ & $52.33 \mathrm{~m}$ \\
\hline $\mathrm{V}_{9} \mathrm{As}_{2}$ & $22.67 \mathrm{r}$ & $35.00 \mathrm{pq}$ & 43.00 no & 47.67 no & 42.00 no \\
\hline $\mathrm{V}_{10} \mathrm{As}_{0}$ & $36.00 \mathrm{gh}$ & $57.00 \mathrm{j}$ & 63.331 & $69.00 \mathrm{jk}$ & 62.331 \\
\hline $\mathrm{V}_{10} \mathrm{As}_{1}$ & $35.00 \mathrm{~g}-\mathrm{i}$ & $56.00 \mathrm{jk}$ & 62.331 & $68.00 \mathrm{jk}$ & 61.331 \\
\hline $\mathrm{V}_{10} \mathrm{As}_{2}$ & $32.67 \mathrm{~h}-\mathrm{j}$ & $41.33 \mathrm{o}$ & $46.67 n$ & $50.00 \mathrm{n}$ & $45.67 \mathrm{n}$ \\
\hline $\mathrm{V}_{11} \mathrm{As}_{0}$ & 42.33 ef & $68.33 \mathrm{f}-\mathrm{i}$ & $77.00 \mathrm{~h}-\mathrm{j}$ & $80.00 \mathrm{gh}$ & $76.00 \mathrm{~h}-\mathrm{j}$ \\
\hline $\mathrm{V}_{11} \mathrm{As}_{1}$ & $41.33 \mathrm{f}$ & $67.33 \mathrm{~g}-\mathrm{i}$ & $76.00 \mathrm{ij}$ & $79.00 \mathrm{hi}$ & $75.00 \mathrm{ij}$ \\
\hline $\mathrm{V}_{11} \mathrm{As}_{2}$ & $39.33 \mathrm{fg}$ & $43.67 \mathrm{o}$ & $47.33 n$ & 48.67 no & $46.33 n$ \\
\hline $\mathrm{V}_{12} \mathrm{As}_{0}$ & $50.00 \mathrm{~b}-\mathrm{d}$ & $68.67 \mathrm{f}-\mathrm{i}$ & $77.33 \mathrm{hi}$ & $80.00 \mathrm{gh}$ & $76.33 \mathrm{hi}$ \\
\hline $\mathrm{V}_{12} \mathrm{As}_{1}$ & $49.00 \mathrm{~cd}$ & $67.67 \mathrm{f}-\mathrm{i}$ & $76.33 \mathrm{ij}$ & $79.00 \mathrm{hi}$ & 75.33 ij \\
\hline $\mathrm{V}_{12} \mathrm{As}_{2}$ & $47.00 \mathrm{de}$ & $54.33 \mathrm{j}-1$ & $58.33 \mathrm{~lm}$ & $59.67 \mathrm{~lm}$ & $57.33 \mathrm{~lm}$ \\
\hline $\mathrm{V}_{13} \mathrm{As}_{0}$ & $29.33 \mathrm{j}-\mathrm{o}$ & 70.33 e-g & $134.0 \mathrm{c}$ & $156.3 \mathrm{~b}$ & $133.0 \mathrm{c}$ \\
\hline $\mathrm{V}_{13} \mathrm{As}_{1}$ & $28.33 \mathrm{j}-\mathrm{q}$ & $69.33 \mathrm{f}-\mathrm{h}$ & $133.0 \mathrm{c}$ & $155.3 \mathrm{~b}$ & $132.0 \mathrm{c}$ \\
\hline $\mathrm{V}_{13} \mathrm{As}_{2}$ & $26.331-\mathrm{r}$ & $50.67 \mathrm{k}-\mathrm{m}$ & $111.7 \mathrm{e}$ & $131.0 \mathrm{~d}$ & $110.7 \mathrm{e}$ \\
\hline $\mathrm{V}_{14} \mathrm{As}_{0}$ & $16.33 \mathrm{~s}$ & $30.00 \mathrm{q}-\mathrm{s}$ & $168.3 \mathrm{a}$ & $187.7 \mathrm{a}$ & $167.3 \mathrm{a}$ \\
\hline $\mathrm{V}_{14} \mathrm{As}_{1}$ & $15.33 \mathrm{~s}$ & 29.00 rs & $167.3 \mathrm{a}$ & $186.7 \mathrm{a}$ & $166.3 \mathrm{a}$ \\
\hline $\mathrm{V}_{14} \mathrm{As}_{2}$ & $13.67 \mathrm{~s}$ & $18.33 \mathrm{t}$ & $140.7 \mathrm{~b}$ & $147.7 \mathrm{c}$ & $139.7 \mathrm{~b}$ \\
\hline SE value & 1.54 & 1.75 & 1.97 & 2.01 & 1.97 \\
\hline CV (\%) & 7.17 & 5.38 & 4.40 & 4.18 & 4.46 \\
\hline
\end{tabular}

In a column means having similar letter (s) are statistically similar and those having dissimilar letter (s) differ significantly by DMRT at 0.05 level of probability 


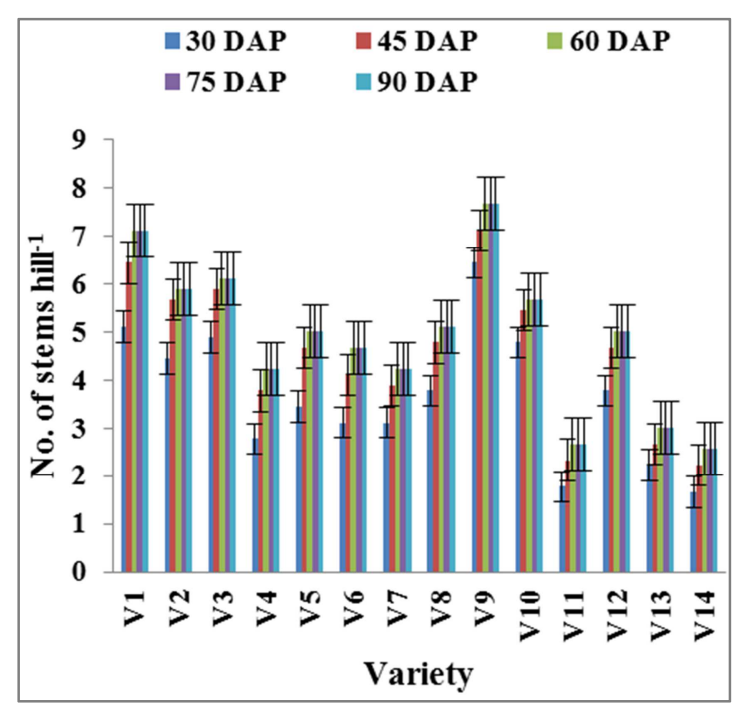

Figure 3. Effect of varieties on number of stems hill ${ }^{1}$ at different growth stages of potato (Vertical bar represents SE value).

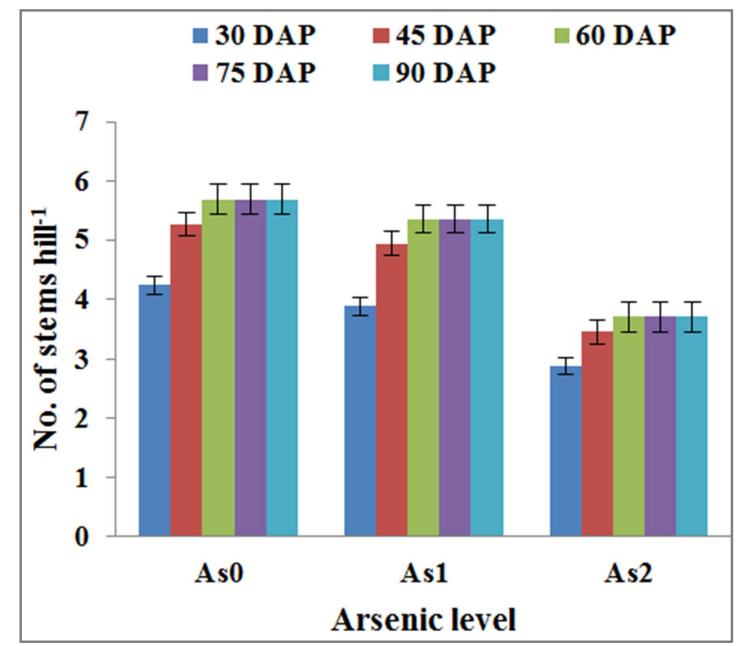

Figure 4. Effect of As levels on number of stems hill ${ }^{-1}$ at different growth stages of potato (Vertical bar represents SE value).

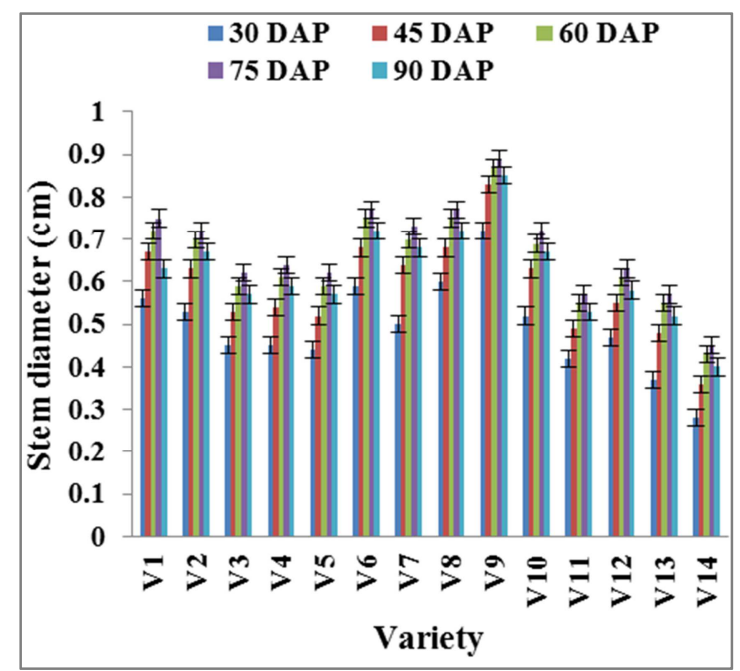

Figure 5. Effect of varieties on stem diameter at different growth stages of potato (Vertical bar represents SE value).

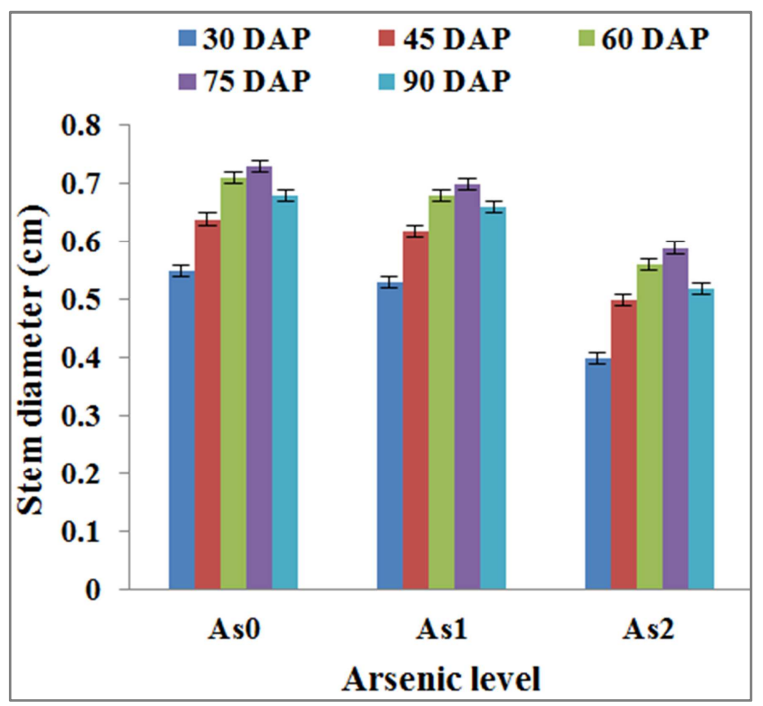

Figure 6. Effect of As levels on stem diameter at different growth stages of potato (Vertical bar represents $S E$ value).

\subsection{Number of Stems Hill ${ }^{1}$}

The number of stems hill ${ }^{-1}$ was significantly varied between the varieties and/or As levels at different growing stages (Figure 3, 4 and Table 4). It was found that number of stems hill $^{-1}$ increased with advancing growing period up to $60 \mathrm{DAP}$ irrespective of varieties and thereafter remained constant (Figure 3). The study referred that 'Felsina' variety produced maximum number of stems hill ${ }^{-1}$; it might be due to varietal characters. On the other hand, from figure 4 it is observed that number of stems hill $^{-1}$ increased with advancing growing period upto 60 DAP irrespective of As levels and thereafter remained constant. Present study showed that number of stems

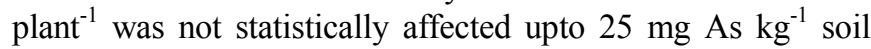
treatment compared to control but at higher concentration (50 $\mathrm{mg} \mathrm{As} \mathrm{kg}{ }^{-1}$ soil) number of stems hill ${ }^{-1}$ significantly decreased. In case of treatment combinations the maximum number of stems hill $^{-1}$ (8.33) was obtained from the combination of 'Felsina' with $\mathrm{As}_{0}$ treatment which was statistically similar (8.00, 8.00, 7.67, 7.33, 7.00, 6.67, 6.67 and 6.67, respectively) with $\mathrm{V}_{1} \mathrm{As}_{0}, \mathrm{~V}_{9} \mathrm{As}_{1}, \mathrm{~V}_{1} \mathrm{As}_{1}, \mathrm{~V}_{3} \mathrm{As}_{0}, \mathrm{~V}_{3} \mathrm{As}_{1}, \mathrm{~V}_{2} \mathrm{As}_{0}, \mathrm{~V}_{10} \mathrm{As}_{0}$, $\mathrm{V}_{9} \mathrm{As}_{2}$ and the minimum (1.67) was recorded from the

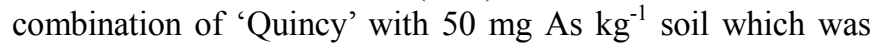
statistically at par $(2.00,2.00,2.67,3.00,3.00,3.00,3.00,3.33$ and 3.33 , respectively) with $\mathrm{V}_{14} \mathrm{As}_{2}, \mathrm{~V}_{13} \mathrm{As}_{2}, \mathrm{~V}_{14} \mathrm{As}_{1}, \mathrm{~V}_{4} \mathrm{As}_{2}$, $\mathrm{V}_{7} \mathrm{As}_{2}, \mathrm{~V}_{14} \mathrm{As}_{0}, \mathrm{~V}_{11} \mathrm{As}_{0}, \mathrm{~V}_{13} \mathrm{As}_{1}$ and $\mathrm{V}_{11} \mathrm{As}_{0}$ at harvesting stage (Table 4).

\subsection{Stem Diameter}

Significant variation was recorded for stem diameter due to different varieties and/or As levels of potato at 30, 45, 60, 75 and 90 DAP (Figure 5, 6 and Table 5). It is revealed that stem diameter increased with advancing growing period upto 75 DAP irrespective of varieties and thereafter decreased (Figure 5). In vegetative stage, potato stems were fleshy and succulent and at later (harvesting) stage it becomes hard and 
slender due to senescence of plant. On the other hand, figure 6 exhibited that stem diameter increased with increasing different growing stages upto 75 DAP irrespective of As levels and thereafter decreased. In vegetative stage potato stems were fleshy and succulent and at later (harvesting) stage it becomes hard and slender due to senescence of plant. In present study $25 \mathrm{mg} \mathrm{As} \mathrm{kg}{ }^{-1}$ soil $\left(\mathrm{As}_{1}\right)$ showed wider and in control $\left(\mathrm{As}_{0}\right)$ performed widest result in terms of diameter of stem. In case of treatment combinations, the widest stem diameter $(0.92 \mathrm{~cm})$ was recorded from the combination of 'Felsina' with $\mathrm{As}_{0}$ which was statistically similar $(0.89 \mathrm{~cm})$ with $\mathrm{V}_{9} \mathrm{As}_{1}$ and the narrowest $(0.31 \mathrm{~cm})$ was recorded from the combination of 'Jam Alu' with $50 \mathrm{mg} \mathrm{As} \mathrm{kg}^{-1}$ soil at harvesting stage (Table 5).

Table 4. Interaction effect of varieties and As levels on number of stems hill ${ }^{-1}$ at different DAP of potato.

\begin{tabular}{|c|c|c|c|c|c|}
\hline \multirow{2}{*}{ Variety $\times$ As level } & \multicolumn{5}{|c|}{ Number of stems hill ${ }^{-1}$ at } \\
\hline & 30 DAP & 45 DAP & 60 DAP & 75 DAP & 90 DAP \\
\hline $\mathrm{V}_{1} \mathrm{As}_{0}$ & $5.67 \mathrm{~b}$ & $7.33 \mathrm{ab}$ & $8.00 \mathrm{ab}$ & $8.00 \mathrm{ab}$ & $8.00 \mathrm{ab}$ \\
\hline $\mathrm{V}_{1} \mathrm{As}_{1}$ & $5.33 \mathrm{bc}$ & $7.00 \mathrm{a}-\mathrm{c}$ & $7.67 \mathrm{a}-\mathrm{c}$ & $7.67 \mathrm{a}-\mathrm{c}$ & $7.67 \mathrm{a}-\mathrm{c}$ \\
\hline $\mathrm{V}_{1} \mathrm{As}_{2}$ & $4.33 \mathrm{c}-\mathrm{f}$ & $5.00 \mathrm{e}-\mathrm{i}$ & $5.67 \mathrm{~d}-\mathrm{h}$ & $5.67 \mathrm{~d}-\mathrm{h}$ & $5.67 \mathrm{~d}-\mathrm{h}$ \\
\hline $\mathrm{V}_{2} \mathrm{As}_{0}$ & $5.00 \mathrm{~b}-\mathrm{d}$ & 6.33 a-e & $6.67 \mathrm{a}-\mathrm{f}$ & $6.67 \mathrm{a}-\mathrm{f}$ & $6.67 \mathrm{a}-\mathrm{f}$ \\
\hline $\mathrm{V}_{2} \mathrm{As}_{1}$ & $4.67 \mathrm{~b}-\mathrm{e}$ & $6.00 \mathrm{~b}-\mathrm{f}$ & $6.33 \mathrm{~b}-\mathrm{g}$ & $6.33 \mathrm{~b}-\mathrm{g}$ & $6.33 \mathrm{~b}-\mathrm{g}$ \\
\hline $\mathrm{V}_{2} \mathrm{As}_{2}$ & $3.67 \mathrm{e}-\mathrm{h}$ & $4.67 \mathrm{f}-\mathrm{j}$ & $4.67 \mathrm{~g}-\mathrm{k}$ & $4.67 \mathrm{~g}-\mathrm{k}$ & $4.67 \mathrm{~g}-\mathrm{k}$ \\
\hline $\mathrm{V}_{3} \mathrm{As}_{0}$ & $5.67 \mathrm{~b}$ & $7.00 \mathrm{a}-\mathrm{c}$ & $7.33 \mathrm{a}-\mathrm{d}$ & $7.33 \mathrm{a}-\mathrm{d}$ & $7.33 \mathrm{a}-\mathrm{d}$ \\
\hline $\mathrm{V}_{3} \mathrm{As}_{1}$ & $5.00 \mathrm{~b}-\mathrm{d}$ & $6.67 \mathrm{a}-\mathrm{d}$ & 7.00 a-e & 7.00 a-e & 7.00 a-e \\
\hline $\mathrm{V}_{3} \mathrm{As}_{2}$ & $4.00 \mathrm{~d}-\mathrm{g}$ & $4.00 \mathrm{~h}-1$ & $4.00 \mathrm{~h}-1$ & $4.00 \mathrm{~h}-1$ & $4.00 \mathrm{~h}-1$ \\
\hline $\mathrm{V}_{4} \mathrm{As}_{0}$ & $3.33 \mathrm{f}-\mathrm{i}$ & $4.33 \mathrm{~g}-\mathrm{k}$ & $5.00 \mathrm{f}-\mathrm{j}$ & $5.00 \mathrm{f}-\mathrm{j}$ & $5.00 \mathrm{f}-\mathrm{j}$ \\
\hline $\mathrm{V}_{4} \mathrm{As}_{1}$ & $3.00 \mathrm{~g}-\mathrm{j}$ & $4.00 \mathrm{~h}-1$ & $4.67 \mathrm{~g}-\mathrm{k}$ & $4.67 \mathrm{~g}-\mathrm{k}$ & $4.67 \mathrm{~g}-\mathrm{k}$ \\
\hline $\mathrm{V}_{4} \mathrm{As}_{2}$ & $2.00 \mathrm{j}-1$ & $3.00 \mathrm{k}-\mathrm{n}$ & $3.00 \mathrm{k}-\mathrm{n}$ & $3.00 \mathrm{k}-\mathrm{n}$ & $3.00 \mathrm{k}-\mathrm{n}$ \\
\hline $\mathrm{V}_{5} \mathrm{As}_{0}$ & $4.00 \mathrm{~d}-\mathrm{g}$ & $5.33 \mathrm{~d}-\mathrm{h}$ & $5.67 \mathrm{~d}-\mathrm{h}$ & $5.67 \mathrm{~d}-\mathrm{h}$ & $5.67 \mathrm{~d}-\mathrm{h}$ \\
\hline $\mathrm{V}_{5} \mathrm{As}_{1}$ & $3.67 \mathrm{e}-\mathrm{h}$ & $5.00 \mathrm{e}-\mathrm{i}$ & $5.33 \mathrm{e}-\mathrm{i}$ & $5.33 \mathrm{e}-\mathrm{i}$ & $5.33 \mathrm{e}-\mathrm{i}$ \\
\hline $\mathrm{V}_{5} \mathrm{As}_{2}$ & $2.67 \mathrm{~h}-\mathrm{j}$ & $3.67 \mathrm{i}-\mathrm{m}$ & 4.00 h-1 & $4.00 \mathrm{~h}-1$ & 4.00 h-1 \\
\hline $\mathrm{V}_{6} \mathrm{As}_{0}$ & 3.67 e-h & $4.67 \mathrm{f}-\mathrm{j}$ & $5.33 \mathrm{e}-\mathrm{i}$ & $5.33 \mathrm{e}-\mathrm{i}$ & $5.33 \mathrm{e}-\mathrm{i}$ \\
\hline $\mathrm{V}_{6} \mathrm{As}_{1}$ & $3.33 \mathrm{f}-\mathrm{i}$ & $4.33 \mathrm{~g}-\mathrm{k}$ & $5.00 \mathrm{f}-\mathrm{j}$ & $5.00 \mathrm{f}-\mathrm{j}$ & $5.00 \mathrm{f}-\mathrm{j}$ \\
\hline $\mathrm{V}_{6} \mathrm{As}_{2}$ & $2.33 \mathrm{i}-\mathrm{k}$ & $3.33 \mathrm{j}-\mathrm{m}$ & 3.67 i-m & $3.67 \mathrm{i}-\mathrm{m}$ & $3.67 \mathrm{i}-\mathrm{m}$ \\
\hline $\mathrm{V}_{7} \mathrm{As}_{0}$ & 3.67 e-h & $4.67 \mathrm{f}-\mathrm{j}$ & $5.00 \mathrm{f}-\mathrm{j}$ & $5.00 \mathrm{f}-\mathrm{j}$ & $5.00 \mathrm{f}-\mathrm{j}$ \\
\hline $\mathrm{V}_{7} \mathrm{As}_{1}$ & $3.33 \mathrm{f}-\mathrm{i}$ & $4.33 \mathrm{~g}-\mathrm{k}$ & $4.67 \mathrm{~g}-\mathrm{k}$ & $4.67 \mathrm{~g}-\mathrm{k}$ & $4.67 \mathrm{~g}-\mathrm{k}$ \\
\hline $\mathrm{V}_{7} \mathrm{As}_{2}$ & $2.33 \mathrm{i}-\mathrm{k}$ & $2.671-0$ & $3.00 \mathrm{k}-\mathrm{n}$ & $3.00 \mathrm{k}-\mathrm{n}$ & $3.00 \mathrm{k}-\mathrm{n}$ \\
\hline $\mathrm{V}_{8} \mathrm{As}_{0}$ & $4.33 \mathrm{c}-\mathrm{f}$ & $5.67 \mathrm{c}-\mathrm{g}$ & $6.00 \mathrm{c}-\mathrm{g}$ & $6.00 \mathrm{c}-\mathrm{g}$ & $6.00 \mathrm{c}-\mathrm{g}$ \\
\hline $\mathrm{V}_{8} \mathrm{As}_{1}$ & $4.00 \mathrm{~d}-\mathrm{g}$ & $5.33 \mathrm{~d}-\mathrm{h}$ & $5.67 \mathrm{~d}-\mathrm{h}$ & $5.67 \mathrm{~d}-\mathrm{h}$ & $5.67 \mathrm{~d}-\mathrm{h}$ \\
\hline $\mathrm{V}_{8} \mathrm{As}_{2}$ & $3.00 \mathrm{~g}-\mathrm{j}$ & $3.33 \mathrm{j}-\mathrm{m}$ & $3.67 \mathrm{i}-\mathrm{m}$ & 3.67 i-m & $3.67 \mathrm{i}-\mathrm{m}$ \\
\hline $\mathrm{V}_{9} \mathrm{As}_{0}$ & $7.00 \mathrm{a}$ & $7.67 \mathrm{a}$ & $8.33 \mathrm{a}$ & $8.33 \mathrm{a}$ & $8.33 \mathrm{a}$ \\
\hline $\mathrm{V}_{9} \mathrm{As}_{1}$ & $6.67 \mathrm{a}$ & $7.33 \mathrm{ab}$ & $8.00 \mathrm{ab}$ & $8.00 \mathrm{ab}$ & $8.00 \mathrm{ab}$ \\
\hline $\mathrm{V}_{9} \mathrm{As}_{2}$ & $5.67 \mathrm{~b}$ & 6.33 a-e & $6.67 \mathrm{a}-\mathrm{f}$ & $6.67 \mathrm{a}-\mathrm{f}$ & $6.67 \mathrm{a}-\mathrm{f}$ \\
\hline $\mathrm{V}_{10} \mathrm{As}_{0}$ & $5.33 \mathrm{bc}$ & $6.33 \mathrm{a}-\mathrm{e}$ & $6.67 \mathrm{a}-\mathrm{f}$ & $6.67 \mathrm{a}-\mathrm{f}$ & $6.67 \mathrm{a}-\mathrm{f}$ \\
\hline $\mathrm{V}_{10} \mathrm{As}_{1}$ & $5.00 \mathrm{~b}-\mathrm{d}$ & $6.00 \mathrm{~b}-\mathrm{f}$ & $6.33 \mathrm{~b}-\mathrm{g}$ & $6.33 \mathrm{~b}-\mathrm{g}$ & $6.33 \mathrm{~b}-\mathrm{g}$ \\
\hline $\mathrm{V}_{10} \mathrm{As}_{2}$ & $4.00 \mathrm{~d}-\mathrm{g}$ & $4.00 \mathrm{~h}-1$ & $4.00 \mathrm{~h}-1$ & $4.00 \mathrm{~h}-1$ & $4.00 \mathrm{~h}-1$ \\
\hline $\mathrm{V}_{11} \mathrm{As}_{0}$ & $2.33 \mathrm{i}-\mathrm{k}$ & $3.00 \mathrm{k}-\mathrm{n}$ & $3.33 \mathrm{j}-\mathrm{n}$ & $3.33 \mathrm{j}-\mathrm{n}$ & $3.33 \mathrm{j}-\mathrm{n}$ \\
\hline $\mathrm{V}_{11} \mathrm{As}_{1}$ & $2.00 \mathrm{j}-1$ & $2.671-0$ & $3.00 \mathrm{k}-\mathrm{n}$ & $3.00 \mathrm{k}-\mathrm{n}$ & $3.00 \mathrm{k}-\mathrm{n}$ \\
\hline $\mathrm{V}_{11} \mathrm{As}_{2}$ & 1.001 & $1.33 \mathrm{o}$ & $1.67 \mathrm{n}$ & $1.67 \mathrm{n}$ & $1.67 \mathrm{n}$ \\
\hline $\mathrm{V}_{12} \mathrm{As}_{0}$ & $4.33 \mathrm{c}-\mathrm{f}$ & $5.33 \mathrm{~d}-\mathrm{h}$ & $5.67 \mathrm{~d}-\mathrm{h}$ & $5.67 \mathrm{~d}-\mathrm{h}$ & $5.67 \mathrm{~d}-\mathrm{h}$ \\
\hline $\mathrm{V}_{12} \mathrm{As}_{1}$ & $4.00 \mathrm{~d}-\mathrm{g}$ & $5.00 \mathrm{e}-\mathrm{i}$ & $5.33 \mathrm{e}-\mathrm{i}$ & $5.33 \mathrm{e}-\mathrm{i}$ & $5.33 \mathrm{e}-\mathrm{i}$ \\
\hline $\mathrm{V}_{12} \mathrm{As}_{2}$ & $3.00 \mathrm{~g}-\mathrm{j}$ & 3.67 i-m & 4.00 h-1 & $4.00 \mathrm{~h}-1$ & 4.00 h-1 \\
\hline $\mathrm{V}_{13} \mathrm{As}_{0}$ & $3.00 \mathrm{~g}-\mathrm{j}$ & $3.33 \mathrm{j}-\mathrm{m}$ & 3.67 i-m & $3.67 \mathrm{i}-\mathrm{m}$ & $3.67 \mathrm{i}-\mathrm{m}$ \\
\hline $\mathrm{V}_{13} \mathrm{As}_{1}$ & $2.33 \mathrm{i}-\mathrm{k}$ & $3.00 \mathrm{k}-\mathrm{n}$ & $3.33 \mathrm{j}-\mathrm{n}$ & $3.33 \mathrm{j}-\mathrm{n}$ & $3.33 \mathrm{j}-\mathrm{n}$ \\
\hline $\mathrm{V}_{13} \mathrm{As}_{2}$ & $1.33 \mathrm{kl}$ & 1.67 no & $2.00 \mathrm{mn}$ & $2.00 \mathrm{mn}$ & $2.00 \mathrm{mn}$ \\
\hline $\mathrm{V}_{14} \mathrm{As}_{0}$ & $2.00 \mathrm{j}-1$ & $2.671-0$ & $3.00 \mathrm{k}-\mathrm{n}$ & $3.00 \mathrm{k}-\mathrm{n}$ & $3.00 \mathrm{k}-\mathrm{n}$ \\
\hline $\mathrm{V}_{14} \mathrm{As}_{1}$ & $2.00 \mathrm{j}-1$ & $2.33 \mathrm{~m}-\mathrm{o}$ & $2.671-n$ & $2.671-n$ & $2.671-n$ \\
\hline $\mathrm{V}_{14} \mathrm{As}_{2}$ & 1.001 & 1.67 no & $2.00 \mathrm{mn}$ & $2.00 \mathrm{mn}$ & $2.00 \mathrm{mn}$ \\
\hline SE value & 0.32 & 0.43 & 0.55 & 0.55 & 0.55 \\
\hline CV (\%) & 15.06 & 16.32 & 19.33 & 19.33 & 19.33 \\
\hline
\end{tabular}

In a column means having similar letter (s) are statistically similar and those having dissimilar letter (s) differ significantly by DMRT at 0.05 level of probability 
Table 5. Interaction effect of varieties and As levels on stem diameter at different DAP of potato.

\begin{tabular}{|c|c|c|c|c|c|}
\hline \multirow{2}{*}{ Variety $\times$ As level } & \multicolumn{5}{|c|}{ Stem diameter $(\mathrm{cm})$ at } \\
\hline & 30 DAP & 45 DAP & 60 DAP & 75 DAP & 90 DAP \\
\hline $\mathrm{V}_{1} \mathrm{As}_{0}$ & 0.62 b-e & $0.73 \mathrm{~b}-\mathrm{d}$ & $0.78 \mathrm{bc}$ & $0.80 \mathrm{~b}-\mathrm{d}$ & 0.69 e-h \\
\hline $\mathrm{V}_{1} \mathrm{As}_{1}$ & $0.60 \mathrm{c}-\mathrm{e}$ & $0.71 \mathrm{~b}-\mathrm{e}$ & $0.76 \mathrm{bc}$ & $0.78 \mathrm{~b}-\mathrm{d}$ & $0.67 \mathrm{f}-\mathrm{i}$ \\
\hline $\mathrm{V}_{1} \mathrm{As}_{2}$ & $0.451-0$ & $0.56 \mathrm{~g}-1$ & 0.64 e-g & $0.67 \mathrm{~g}-\mathrm{i}$ & $0.54 \mathrm{pq}$ \\
\hline $\mathrm{V}_{2} \mathrm{As}_{0}$ & $0.59 \mathrm{c}-\mathrm{f}$ & $0.70 \mathrm{~b}-\mathrm{e}$ & $0.74 \mathrm{bc}$ & $0.76 \mathrm{~b}-\mathrm{d}$ & $0.72 \mathrm{c}-\mathrm{f}$ \\
\hline $\mathrm{V}_{2} \mathrm{As}_{1}$ & $0.57 \mathrm{~d}-\mathrm{g}$ & $0.67 \mathrm{de}$ & $0.72 \mathrm{~cd}$ & $0.74 \mathrm{~d}-\mathrm{f}$ & $0.70 \mathrm{~d}-\mathrm{g}$ \\
\hline $\mathrm{V}_{2} \mathrm{As}_{2}$ & $0.43 \mathrm{~m}-\mathrm{p}$ & $0.54 \mathrm{i}-1$ & $0.62 \mathrm{e}-\mathrm{g}$ & $0.65 \mathrm{~g}-\mathrm{k}$ & $0.59 \mathrm{j}-\mathrm{p}$ \\
\hline $\mathrm{V}_{3} \mathrm{As}_{0}$ & $0.51 \mathrm{~h}-1$ & $0.60 \mathrm{~g}-\mathrm{i}$ & $0.65 \mathrm{ef}$ & $0.67 \mathrm{~g}-\mathrm{i}$ & $0.63 \mathrm{i}-\mathrm{m}$ \\
\hline $\mathrm{V}_{3} \mathrm{As}_{1}$ & 0.48 i-m & $0.58 \mathrm{~g}-\mathrm{k}$ & $0.63 \mathrm{e}-\mathrm{g}$ & $0.65 \mathrm{~g}-\mathrm{k}$ & $0.61 \mathrm{i}-\mathrm{o}$ \\
\hline $\mathrm{V}_{3} \mathrm{As}_{2}$ & $0.35 \mathrm{r}-\mathrm{t}$ & 0.43 no & $0.50 \mathrm{~h}$ & $0.53 \mathrm{~m}-\mathrm{o}$ & $0.47 \mathrm{rs}$ \\
\hline $\mathrm{V}_{4} \mathrm{As}_{0}$ & $0.51 \mathrm{i}-1$ & $0.61 \mathrm{fg}$ & $0.67 \mathrm{de}$ & $0.69 \mathrm{f}-\mathrm{h}$ & $0.65 \mathrm{~g}-\mathrm{j}$ \\
\hline $\mathrm{V}_{4} \mathrm{As}_{1}$ & $0.49 \mathrm{i}-\mathrm{m}$ & $0.58 \mathrm{~g}-\mathrm{j}$ & $0.65 \mathrm{ef}$ & $0.67 \mathrm{~g}-\mathrm{i}$ & $0.63 \mathrm{i}-\mathrm{m}$ \\
\hline $\mathrm{V}_{4} \mathrm{As}_{2}$ & $0.35 \mathrm{q}-\mathrm{s}$ & 0.44 no & $0.52 \mathrm{~h}$ & $0.55 \mathrm{~lm}$ & $0.49 \mathrm{qr}$ \\
\hline $\mathrm{V}_{5} \mathrm{As}_{0}$ & $0.50 \mathrm{i}-1$ & $0.58 \mathrm{~g}-\mathrm{j}$ & $0.65 \mathrm{ef}$ & $0.67 \mathrm{~g}-\mathrm{i}$ & $0.63 \mathrm{i}-\mathrm{m}$ \\
\hline $\mathrm{V}_{5} \mathrm{As}_{1}$ & $0.47 \mathrm{j}-\mathrm{n}$ & $0.56 \mathrm{~g}-1$ & $0.63 \mathrm{e}-\mathrm{g}$ & $0.65 \mathrm{~g}-\mathrm{k}$ & $0.61 \mathrm{j}-\mathrm{o}$ \\
\hline $\mathrm{V}_{5} \mathrm{As}_{2}$ & $0.34 \mathrm{r}-\mathrm{t}$ & 0.43 no & $0.51 \mathrm{~h}$ & $0.54 \mathrm{mn}$ & $0.48 \mathrm{rs}$ \\
\hline $\mathrm{V}_{6} \mathrm{As}_{0}$ & $0.65 \mathrm{bc}$ & $0.74 \mathrm{bc}$ & $0.80 \mathrm{~b}$ & $0.82 \mathrm{bc}$ & $0.78 \mathrm{bc}$ \\
\hline $\mathrm{V}_{6} \mathrm{As}_{1}$ & $0.63 \mathrm{~b}-\mathrm{d}$ & $0.72 \mathrm{~b}$-e & $0.77 \mathrm{bc}$ & $0.79 \mathrm{~b}-\mathrm{d}$ & $0.75 \mathrm{~b}-\mathrm{d}$ \\
\hline $\mathrm{V}_{6} \mathrm{As}_{2}$ & $0.49 \mathrm{i}-1$ & $0.58 \mathrm{~g}-\mathrm{k}$ & $0.67 \mathrm{de}$ & $0.70 \mathrm{e}-\mathrm{g}$ & 0.64 h-1 \\
\hline $\mathrm{V}_{7} \mathrm{As}_{0}$ & $0.54 \mathrm{f}-\mathrm{i}$ & $0.69 \mathrm{~b}-\mathrm{e}$ & $0.76 \mathrm{bc}$ & $0.78 \mathrm{~b}-\mathrm{d}$ & 0.74 b-e \\
\hline $\mathrm{V}_{7} \mathrm{As}_{1}$ & $0.52 \mathrm{~g}-\mathrm{k}$ & $0.67 \mathrm{de}$ & $0.73 \mathrm{c}$ & $0.76 \mathrm{~cd}$ & $0.71 \mathrm{~d}-\mathrm{f}$ \\
\hline $\mathrm{V}_{7} \mathrm{As}_{2}$ & 0.46 k-o & $0.56 \mathrm{~g}-1$ & $0.61 \mathrm{e}-\mathrm{g}$ & 0.64 g-k & $0.58 \mathrm{k-p}$ \\
\hline $\mathrm{V}_{8} \mathrm{As}_{0}$ & $0.67 \mathrm{~b}$ & $0.73 \mathrm{bc}$ & $0.80 \mathrm{~b}$ & $0.82 \mathrm{~b}$ & $0.78 \mathrm{~b}$ \\
\hline $\mathrm{V}_{8} \mathrm{As}_{1}$ & $0.65 \mathrm{bc}$ & $0.71 \mathrm{~b}$-e & $0.78 \mathrm{bc}$ & $0.80 \mathrm{~b}-\mathrm{d}$ & $0.76 \mathrm{~b}-\mathrm{d}$ \\
\hline $\mathrm{V}_{8} \mathrm{As}_{2}$ & $0.48 \mathrm{i}-\mathrm{n}$ & $0.61 \mathrm{fg}$ & $0.65 \mathrm{ef}$ & $0.68 \mathrm{gh}$ & $0.62 \mathrm{i}-\mathrm{m}$ \\
\hline $\mathrm{V}_{9} \mathrm{As}_{0}$ & $0.78 \mathrm{a}$ & $0.88 \mathrm{a}$ & $0.94 \mathrm{a}$ & $0.96 \mathrm{a}$ & $0.92 \mathrm{a}$ \\
\hline $\mathrm{V}_{9} \mathrm{As}_{1}$ & $0.76 \mathrm{a}$ & $0.86 \mathrm{a}$ & $0.91 \mathrm{a}$ & $0.93 \mathrm{a}$ & $0.89 \mathrm{a}$ \\
\hline $\mathrm{V}_{9} \mathrm{As}_{2}$ & $0.62 \mathrm{~b}-\mathrm{e}$ & $0.75 \mathrm{~b}$ & $0.76 \mathrm{bc}$ & $0.79 \mathrm{~b}-\mathrm{d}$ & $0.73 \mathrm{~b}-\mathrm{e}$ \\
\hline $\mathrm{V}_{10} \mathrm{As}_{0}$ & $0.59 \mathrm{~d}-\mathrm{f}$ & $0.68 c-e$ & $0.75 \mathrm{bc}$ & $0.77 \mathrm{~b}-\mathrm{d}$ & 0.73 b-e \\
\hline $\mathrm{V}_{10} \mathrm{As}_{1}$ & $0.57 \mathrm{e}-\mathrm{h}$ & $0.66 \mathrm{ef}$ & $0.73 \mathrm{c}$ & $0.75 \mathrm{de}$ & $0.71 \mathrm{~d}-\mathrm{f}$ \\
\hline $\mathrm{V}_{10} \mathrm{As}_{2}$ & $0.42 \mathrm{n}-\mathrm{p}$ & $0.54 \mathrm{~h}-1$ & $0.60 \mathrm{fg}$ & $0.63 \mathrm{~h}-\mathrm{k}$ & $0.57 \mathrm{~m}-\mathrm{p}$ \\
\hline $\mathrm{V}_{11} \mathrm{As}_{0}$ & $0.48 \mathrm{i}-\mathrm{n}$ & $0.54 \mathrm{~h}-1$ & $0.60 \mathrm{fg}$ & $0.62 \mathrm{i}-\mathrm{k}$ & $0.581-p$ \\
\hline $\mathrm{V}_{11} \mathrm{As}_{1}$ & 0.46 k-o & $0.52 \mathrm{k}-\mathrm{m}$ & $0.58 \mathrm{~g}$ & $0.60 \mathrm{j}-1$ & $0.56 n-p$ \\
\hline $\mathrm{V}_{11} \mathrm{As}_{2}$ & $0.33 \mathrm{r}-\mathrm{t}$ & $0.41 \mathrm{o}$ & $0.47 \mathrm{~h}$ & $0.49 \mathrm{~m}-\mathrm{o}$ & $0.44 \mathrm{rs}$ \\
\hline $\mathrm{V}_{12} \mathrm{As}_{0}$ & $0.53 \mathrm{~g}-\mathrm{j}$ & $0.60 \mathrm{gh}$ & $0.66 \mathrm{e}$ & $0.68 \mathrm{gh}$ & $0.64 \mathrm{~g}-\mathrm{k}$ \\
\hline $\mathrm{V}_{12} \mathrm{As}_{1}$ & $0.51 \mathrm{i}-1$ & $0.58 \mathrm{~g}-\mathrm{k}$ & 0.64 e-g & $0.66 \mathrm{~g}-\mathrm{j}$ & $0.62 \mathrm{i}-\mathrm{n}$ \\
\hline $\mathrm{V}_{12} \mathrm{As}_{2}$ & 0.38 p-r & $0.47 \mathrm{mn}$ & $0.52 \mathrm{~h}$ & $0.551-n$ & $0.49 \mathrm{rs}$ \\
\hline $\mathrm{V}_{13} \mathrm{As}_{0}$ & $0.43 \mathrm{~m}-\mathrm{p}$ & $0.53 \mathrm{j}-1$ & $0.61 \mathrm{e}-\mathrm{g}$ & $0.62 \mathrm{i}-\mathrm{k}$ & $0.581-p$ \\
\hline $\mathrm{V}_{13} \mathrm{As}_{1}$ & $0.40 \mathrm{o}-\mathrm{q}$ & $0.51 \mathrm{~lm}$ & $0.58 \mathrm{~g}$ & $0.60 \mathrm{kl}$ & 0.56 op \\
\hline $\mathrm{V}_{13} \mathrm{As}_{2}$ & $0.29 \mathrm{t}$ & $0.40 \mathrm{o}$ & $0.46 \mathrm{~h}$ & 0.49 no & $0.43 \mathrm{~s}$ \\
\hline $\mathrm{V}_{14} \mathrm{As}_{0}$ & $0.33 \mathrm{r}-\mathrm{t}$ & 0.41 no & $0.48 \mathrm{~h}$ & $0.50 \mathrm{~m}-\mathrm{o}$ & $0.46 \mathrm{rs}$ \\
\hline $\mathrm{V}_{14} \mathrm{As}_{1}$ & $0.30 \mathrm{st}$ & $0.39 \mathrm{o}$ & $0.46 \mathrm{~h}$ & 0.48 o & $0.44 \mathrm{rs}$ \\
\hline $\mathrm{V}_{14} \mathrm{As}_{2}$ & $0.20 \mathrm{u}$ & $0.28 \mathrm{p}$ & $0.34 \mathrm{i}$ & $0.37 \mathrm{p}$ & $0.31 \mathrm{t}$ \\
\hline SE value & 0.018 & 0.018 & 0.018 & 0.018 & 0.018 \\
\hline CV (\%) & 7.45 & 4.30 & 4.54 & 4.54 & 3.94 \\
\hline
\end{tabular}

In a column means having similar letter (s) are statistically similar and those having dissimilar letter (s) differ significantly by DMRT at 0.05 level of probability 


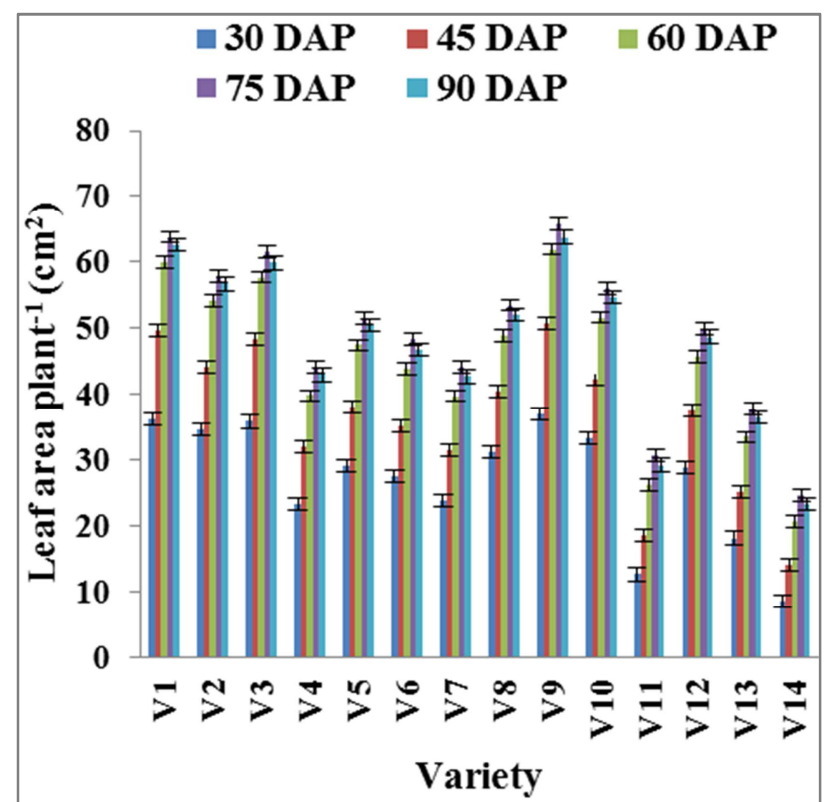

Figure 7. Effect of varieties on leaf area plant ${ }^{1}$ at different growth stages of potato (Vertical bar represents $S E$ value).

\subsection{Leaf Area Plant ${ }^{1}$}

Varieties and/or As levels significantly influenced the leaf area of potato at $30,45,60,75$ and $90 \mathrm{DAP}$ (Figure 7, 8 and Table 6). Figure 7 showed that leaf area increased with advancing growing period upto 75 DAP irrespective of varieties and thereafter decreased due to senescence of plant. This study referred that the potato variety 'Felsina' exposed best result in terms of leaf area. On the contrary, leaf area increased with advancing growing period upto 75 DAP irrespective of As levels and thereafter decreased due to senescence of plant (Figure 8). Juzl and Stefl [35] reported that leaf area index of potato plants decreased significantly with the increasing levels of As in irrigated water and soil but the present study showed that leaf area was not statistically affected up to $25 \mathrm{mg} \mathrm{As} \mathrm{kg}^{-1}$ soil compared to control but at higher concentration $\left(50 \mathrm{mg}\right.$ As $\mathrm{kg}^{-1}$ soil) treatment significantly decreased leaf area. In case of treatment combinations Table 6 exhibited that, the highest leaf area plant $^{-1}\left(67.11 \mathrm{~cm}^{2}\right)$ was obtained from the combination of 'Felsina' with $\mathrm{As}_{0}$ treatment which was statistically at par (66.95, 66.03 and $65.89 \mathrm{~cm}^{2}$, respectively) with $\mathrm{V}_{9} \mathrm{As}_{1}$, $\mathrm{V}_{1} \mathrm{As}_{0}, \mathrm{~V}_{1} \mathrm{As}_{1}$ and the lowest $\left(18.12 \mathrm{~cm}^{2}\right)$ was recorded from

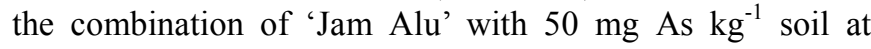
harvesting stage. Finally, in this study it was found that 'Felsina' cultivated with $25 \mathrm{mg} \mathrm{As} \mathrm{kg}^{-1}\left(\mathrm{As}_{1}\right)$ soil showed better and with control $\left(\mathrm{As}_{0}\right)$ performed best result in terms of leaf area plant ${ }^{-1}$.

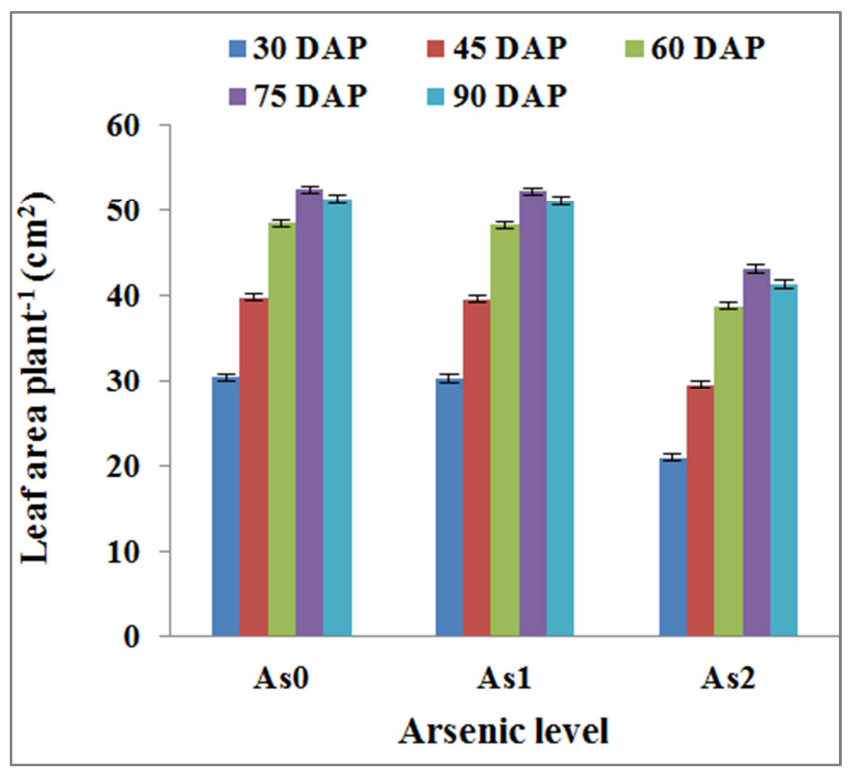

Figure 8. Effect of As levels on leaf area plant ${ }^{1}$ at different growth stages of potato (Vertical bar represents $S E$ value).

Table 6. Interaction effect of varieties and As levels on leaf area plant ${ }^{1}$ at different DAP of potato.

\begin{tabular}{|c|c|c|c|c|c|}
\hline \multirow{2}{*}{ Variety $\times$ As level } & \multicolumn{5}{|c|}{ Leaf area plant ${ }^{-1}\left(\mathrm{~cm}^{2}\right)$ at } \\
\hline & 30 DAP & 45 DAP & 60 DAP & 75 DAP & 90 DAP \\
\hline $\mathrm{V}_{1} \mathrm{As}_{0}$ & $39.25 \mathrm{a}-\mathrm{c}$ & $53.24 \mathrm{a}$ & $63.03 \mathrm{ab}$ & $67.25 \mathrm{ab}$ & $66.03 \mathrm{ab}$ \\
\hline $\mathrm{V}_{1} \mathrm{As}_{1}$ & 39.14 a-c & $53.02 \mathrm{a}$ & $62.80 \mathrm{ab}$ & $67.07 \mathrm{ab}$ & $65.89 \mathrm{a}-\mathrm{c}$ \\
\hline $\mathrm{V}_{1} \mathrm{As}_{2}$ & $30.17 \mathrm{f}-\mathrm{h}$ & $43.17 \mathrm{de}$ & $53.99 \mathrm{~d}-\mathrm{f}$ & $57.04 \mathrm{de}$ & $55.97 \mathrm{fg}$ \\
\hline $\mathrm{V}_{2} \mathrm{As}_{0}$ & $37.81 \mathrm{a}-\mathrm{c}$ & $47.68 \mathrm{~b}$ & $57.29 \mathrm{c}$ & $61.27 \mathrm{c}$ & $60.17 \mathrm{de}$ \\
\hline $\mathrm{V}_{2} \mathrm{As}_{1}$ & $37.64 \mathrm{a}-\mathrm{c}$ & $47.48 \mathrm{~b}$ & $57.06 \mathrm{c}$ & $61.06 \mathrm{c}$ & $59.97 \mathrm{de}$ \\
\hline $\mathrm{V}_{2} \mathrm{As}_{2}$ & $28.64 \mathrm{~g}-\mathrm{j}$ & $37.42 \mathrm{gh}$ & $48.19 \mathrm{ij}$ & $51.13 \mathrm{gh}$ & $50.07 \mathrm{ij}$ \\
\hline $\mathrm{V}_{3} \mathrm{As}_{0}$ & 38.97 a-c & $51.94 \mathrm{a}$ & $60.94 \mathrm{~b}$ & $65.04 \mathrm{~b}$ & $63.14 b c$ \\
\hline $\mathrm{V}_{3} \mathrm{As}_{1}$ & $38.80 \mathrm{a}-\mathrm{c}$ & $51.73 \mathrm{a}$ & $60.63 \mathrm{~b}$ & $64.87 \mathrm{~b}$ & $62.93 \mathrm{~cd}$ \\
\hline $\mathrm{V}_{3} \mathrm{As}_{2}$ & $29.87 \mathrm{f}-\mathrm{i}$ & 41.72 ef & $51.65 \mathrm{f}-\mathrm{h}$ & 54.68 ef & $53.64 \mathrm{gh}$ \\
\hline $\mathrm{V}_{4} \mathrm{As}_{0}$ & $27.05 \mathrm{i}-\mathrm{k}$ & $34.99 \mathrm{hi}$ & $42.881-n$ & $46.91 \mathrm{j}$ & $45.91 \mathrm{kl}$ \\
\hline $\mathrm{V}_{4} \mathrm{As}_{1}$ & $26.91 \mathrm{i}-\mathrm{k}$ & $34.76 \mathrm{hi}$ & $42.661-n$ & $46.74 \mathrm{j}$ & $45.74 \mathrm{kl}$ \\
\hline $\mathrm{V}_{4} \mathrm{As}_{2}$ & $16.20 \mathrm{~m}$ & $26.14 \mathrm{mn}$ & $33.97 \mathrm{q}$ & $39.06 \mathrm{~lm}$ & 37.34 op \\
\hline $\mathrm{V}_{5} \mathrm{As}_{0}$ & 32.83 ef & 41.59 ef & $50.57 \mathrm{~g}-\mathrm{i}$ & $54.33 \mathrm{f}$ & $53.72 \mathrm{gh}$ \\
\hline $\mathrm{V}_{5} \mathrm{As}_{1}$ & 32.56 ef & 41.41 ef & $50.35 \mathrm{hi}$ & $54.14 \mathrm{f}$ & $53.52 \mathrm{gh}$ \\
\hline $\mathrm{V}_{5} \mathrm{As}_{2}$ & 21.841 & $31.62 \mathrm{jk}$ & $41.52 \mathrm{mn}$ & $46.12 \mathrm{j}$ & $44.13 \mathrm{~lm}$ \\
\hline
\end{tabular}




\begin{tabular}{|c|c|c|c|c|c|}
\hline \multirow{2}{*}{ Variety $\times$ As level } & \multicolumn{5}{|c|}{ Leaf area plant ${ }^{-1}\left(\mathrm{~cm}^{2}\right)$ at } \\
\hline & 30 DAP & 45 DAP & 60 DAP & 75 DAP & 90 DAP \\
\hline $\mathrm{V}_{6} \mathrm{As}_{0}$ & $31.25 \mathrm{fg}$ & $39.18 \mathrm{fg}$ & $47.40 \mathrm{jk}$ & $51.34 \mathrm{gh}$ & $50.21 \mathrm{ij}$ \\
\hline $\mathrm{V}_{6} \mathrm{As}_{1}$ & $31.12 \mathrm{fg}$ & $38.99 \mathrm{fg}$ & $47.20 \mathrm{jk}$ & $51.20 \mathrm{gh}$ & $50.04 \mathrm{ij}$ \\
\hline $\mathrm{V}_{6} \mathrm{As}_{2}$ & 20.311 & $27.11 \mathrm{~lm}$ & $37.27 \mathrm{p}$ & $42.45 \mathrm{k}$ & 40.16 no \\
\hline $\mathrm{V}_{7} \mathrm{As}_{0}$ & $27.87 \mathrm{~h}-\mathrm{j}$ & $35.64 \mathrm{hi}$ & $43.53 \mathrm{~lm}$ & $47.52 \mathrm{ij}$ & $46.53 \mathrm{kl}$ \\
\hline $\mathrm{V}_{7} \mathrm{As}_{1}$ & $27.39 \mathrm{~h}-\mathrm{k}$ & $35.47 \mathrm{hi}$ & $43.36 \mathrm{~lm}$ & $47.33 \mathrm{ij}$ & $46.43 \mathrm{kl}$ \\
\hline $\mathrm{V}_{7} \mathrm{As}_{2}$ & $16.73 \mathrm{~m}$ & 23.63 no & $32.55 \mathrm{q}$ & $37.41 \mathrm{~m}$ & $35.39 \mathrm{p}$ \\
\hline $\mathrm{V}_{8} \mathrm{As}_{0}$ & 34.72 de & $44.27 \mathrm{c}-\mathrm{e}$ & 53.14 ef & $57.29 \mathrm{de}$ & $56.12 \mathrm{fg}$ \\
\hline $\mathrm{V}_{8} \mathrm{As}_{1}$ & $34.58 \mathrm{de}$ & $44.04 \mathrm{c}-\mathrm{e}$ & $52.97 \mathrm{e}-\mathrm{g}$ & $57.13 \mathrm{de}$ & $55.93 \mathrm{fg}$ \\
\hline $\mathrm{V}_{8} \mathrm{As}_{2}$ & $24.63 \mathrm{k}$ & $33.24 \mathrm{ij}$ & 40.68 no & $46.14 j$ & $44.16 \mathrm{~lm}$ \\
\hline $\mathrm{V}_{9} \mathrm{As}_{0}$ & 40.19 a & $54.22 \mathrm{a}$ & $65.01 \mathrm{a}$ & $69.24 \mathrm{a}$ & $67.11 \mathrm{a}$ \\
\hline $\mathrm{V}_{9} \mathrm{As}_{1}$ & $40.00 \mathrm{ab}$ & $54.05 \mathrm{a}$ & $64.80 \mathrm{a}$ & $69.08 \mathrm{a}$ & $66.95 \mathrm{a}$ \\
\hline $\mathrm{V}_{9} \mathrm{As}_{2}$ & $31.08 \mathrm{fg}$ & $44.14 \mathrm{c}-\mathrm{e}$ & $55.98 \mathrm{~cd}$ & $58.98 \mathrm{~cd}$ & 57.34 ef \\
\hline $\mathrm{V}_{10} \mathrm{As}_{0}$ & $36.92 \mathrm{~b}-\mathrm{d}$ & $46.13 \mathrm{bc}$ & $55.10 \mathrm{c}-\mathrm{e}$ & $59.19 \mathrm{~cd}$ & 57.95 ef \\
\hline $\mathrm{V}_{10} \mathrm{As}_{1}$ & $36.77 \mathrm{~cd}$ & 45.94 b-d & $54.94 \mathrm{c}-\mathrm{e}$ & $59.05 \mathrm{~cd}$ & 57.81 ef \\
\hline $\mathrm{V}_{10} \mathrm{As}_{2}$ & $26.74 \mathrm{jk}$ & 34.88 hi & $44.96 \mathrm{kl}$ & $49.90 \mathrm{hi}$ & $48.26 \mathrm{jk}$ \\
\hline $\mathrm{V}_{11} \mathrm{As}_{0}$ & $14.98 \mathrm{~m}$ & $21.07 \mathrm{o}$ & $29.07 \mathrm{r}$ & $33.17 n$ & $32.05 \mathrm{q}$ \\
\hline $\mathrm{V}_{11} \mathrm{As}_{1}$ & $14.93 \mathrm{n}$ & $20.90 \mathrm{o}$ & $28.89 \mathrm{r}$ & $33.00 \mathrm{n}$ & $31.92 \mathrm{q}$ \\
\hline $\mathrm{V}_{11} \mathrm{As}_{2}$ & 8.18 op & $13.88 \mathrm{q}$ & $20.86 \mathrm{t}$ & $25.74 \mathrm{o}$ & $23.86 \mathrm{~s}$ \\
\hline $\mathrm{V}_{12} \mathrm{As}_{0}$ & 32.52 ef & 41.48 ef & $49.54 \mathrm{~h}-\mathrm{j}$ & $53.43 \mathrm{fg}$ & $52.50 \mathrm{hi}$ \\
\hline $\mathrm{V}_{12} \mathrm{As}_{1}$ & 32.38 ef & 41.26 ef & $49.32 \mathrm{~h}-\mathrm{j}$ & $53.24 \mathrm{fg}$ & $52.41 \mathrm{hi}$ \\
\hline $\mathrm{V}_{12} \mathrm{As}_{2}$ & 21.701 & $29.87 \mathrm{kl}$ & 38.42 op & $43.29 \mathrm{k}$ & $41.41 \mathrm{mn}$ \\
\hline $\mathrm{V}_{13} \mathrm{As}_{0}$ & 21.621 & $29.22 \mathrm{kl}$ & $37.27 p$ & $41.37 \mathrm{kl}$ & 40.34 no \\
\hline $\mathrm{V}_{13} \mathrm{As}_{1}$ & 21.541 & $29.06 \mathrm{kl}$ & $37.12 p$ & $41.21 \mathrm{kl}$ & 40.27 no \\
\hline $\mathrm{V}_{13} \mathrm{As}_{2}$ & $11.49 \mathrm{n}$ & $17.30 \mathrm{p}$ & $26.30 \mathrm{~s}$ & $31.09 \mathrm{n}$ & $29.02 \mathrm{r}$ \\
\hline $\mathrm{V}_{14} \mathrm{As}_{0}$ & 10.16 no & $16.20 \mathrm{pq}$ & $23.02 \mathrm{t}$ & $27.04 \mathrm{o}$ & $26.04 \mathrm{~s}$ \\
\hline $\mathrm{V}_{14} \mathrm{As}_{1}$ & 10.11 no & $16.09 \mathrm{pq}$ & $22.90 \mathrm{t}$ & 26.86 o & $25.91 \mathrm{~s}$ \\
\hline $\mathrm{V}_{14} \mathrm{As}_{2}$ & $5.58 \mathrm{p}$ & $10.14 \mathrm{r}$ & $16.31 \mathrm{u}$ & $20.19 \mathrm{p}$ & $18.12 \mathrm{t}$ \\
\hline SE value & 0.94 & 0.92 & 0.84 & 0.90 & 1.00 \\
\hline CV (\%) & 5.99 & 4.41 & 3.21 & 3.16 & 3.62 \\
\hline
\end{tabular}

In a column means having similar letter (s) are statistically similar and those having dissimilar letter (s) differ significantly by DMRT at 0.05 level of probability

\subsection{Chlorophyll Content of Leaves}

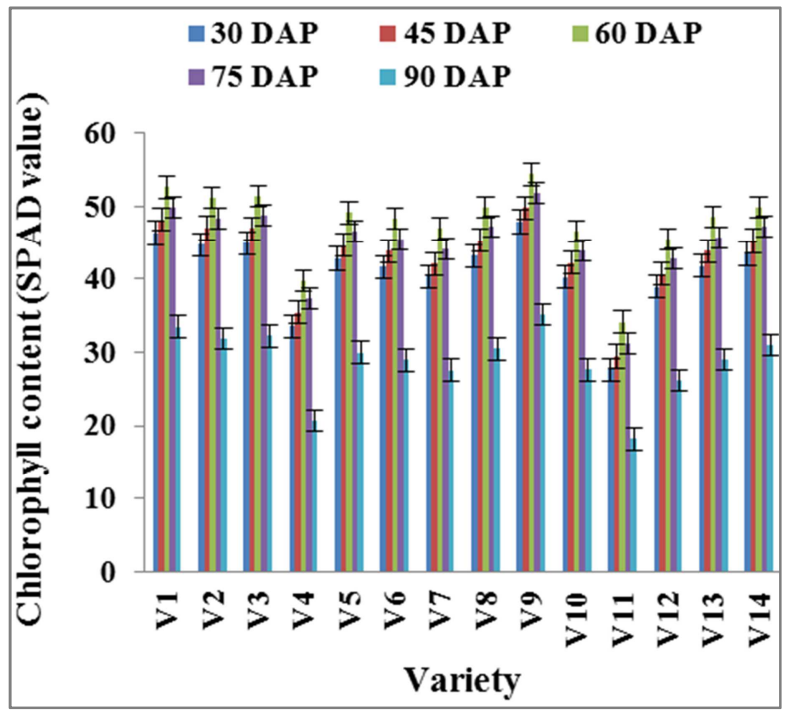

Figure 9. Effect of varieties on chlorophyll content of leaves at different growth stages of potato leaf (Vertical bar represents SE value)

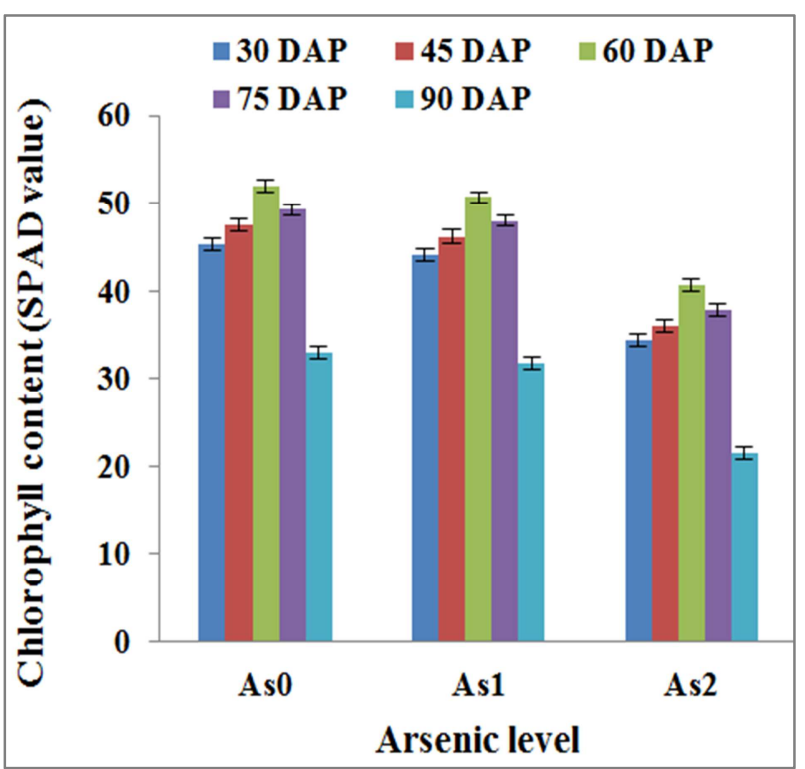

Figure 10. Effect of As levels on chlorophyll content of leaves at different growth stages of potato leaf (Vertical bar represents SE value) 
Chlorophyll content (SPAD value) of potato leaves were significantly affected by the varieties and/or As levels at different growing stages (Figure 9, 10 and Table 7). Figure 9 showed that chlorophyll content (SPAD value) increased with advancing growing period upto 60 DAP irrespective of varieties and thereafter decreased due to yellowing of leaves. Potato varieties used in the study differed in chlorophyll content reading, like observed by many other workers [36, 37]. On the other hand, Figure 10 showed that chlorophyll content (SPAD value) increased with increasing growing period upto 60 DAP irrespective of As levels and thereafter decreased due to yellowing of leaves. Nitrogen is the core component of chlorophyll molecule and thus, its content in leaf is directly correlated with chlorophyll content. It is revealed that higher soil As concentrations decrease the nitrogen content in garden pea [38] and silver bet [39]. Miteva and Merakchiyska [40] reported that As concentrations of $25 \mathrm{mg} \mathrm{kg}^{-1}$ soil did not have negative effect on the photosynthetic process in bean plants (Phaseolus vulgaris L.), while the higher doses (50 and $100 \mathrm{mg}$ of As kg${ }^{1}$ soil) inhibit the photosynthesis by 42 and $32 \%$, respectively. Increased As concentrations caused an alternation of the chloroplast shape, manifested in its rounding and shortening of the longitudinal axis of plant cell. Other manifestations are concaving membrane, bending and partial destruction as well as changes in the accumulation and flow of assimilates which results in the decrease of chlorophyll content in potato leaf. Thus, it was expected that the higher soil As concentrations may also decrease nitrogen content in potato plant which may also cause the decrease of chlorophyll content. The results of the present experiment revealed that chlorophyll content of potato leaves were not statistically affected upto $25 \mathrm{mg} \mathrm{As} \mathrm{kg}^{-1}$ soil treatment compared to control but at higher concentration (50 mg As $\mathrm{kg}^{-1}$ soil) treatment chlorophyll content significantly decreased. In case of treatment combinations, the maximum chlorophyll content (39.48 SPAD value) was recorded from the 'Felsina' with $\mathrm{As}_{0}$ treatment which was statistically similar $(38.24,37.73,36.50$, $36.42,36.13,35.19,35.06,34.90$ and $34.80 \mathrm{SPAD}$ value, respectively) with $\mathrm{V}_{9} \mathrm{As}_{1}, \mathrm{~V}_{1} \mathrm{As}_{0}, \mathrm{~V}_{1} \mathrm{As}_{1}, \mathrm{~V}_{3} \mathrm{As}_{0}, \mathrm{~V}_{2} \mathrm{As}_{0}$, $\mathrm{V}_{3} \mathrm{As}_{1}, \mathrm{~V}_{14} \mathrm{As}_{0}, \mathrm{~V}_{2} \mathrm{As}_{1}, \mathrm{~V}_{8} \mathrm{As}_{0}$ and the minimum (18.21 SPAD value) was recorded from the combination of 'Quincy' with $50 \mathrm{mg} \mathrm{As} \mathrm{kg}^{-1}$ soil at harvesting stage (Table 7). The variation in total chlorophyll content may be a good indicator of stress in plants that have been caused by environmental factors $[41,42]$.

Table 7. Interaction effect of varieties and As levels on chlorophyll content of leaf at different DAP and above ground stem dry matter content of potato

\begin{tabular}{|c|c|c|c|c|c|c|}
\hline \multirow{2}{*}{$\begin{array}{l}\text { Variety } \times \text { As } \\
\text { level }\end{array}$} & \multicolumn{5}{|c|}{ Chlorophyll content (SPAD value) of leaves at } & \multirow{2}{*}{$\begin{array}{l}\text { Above ground stem dry } \\
\text { matter content }(\%)\end{array}$} \\
\hline & 30 DAP & 45 DAP & 60 DAP & 75 DAP & 90 DAP & \\
\hline $\mathrm{V}_{1} \mathrm{As}_{0}$ & $50.27 \mathrm{ab}$ & $52.38 \mathrm{ab}$ & 56.84 a-c & 54.18 a-c & $37.73 \mathrm{a}-\mathrm{c}$ & $17.16 \mathrm{e}-\mathrm{h}$ \\
\hline $\mathrm{V}_{1} \mathrm{As}_{1}$ & $49.03 \mathrm{a}-\mathrm{c}$ & $51.14 \mathrm{a}-\mathrm{c}$ & $55.60 \mathrm{a}-\mathrm{d}$ & 52.94 a-d & $36.50 \mathrm{a}-\mathrm{d}$ & $16.75 \mathrm{f}-\mathrm{j}$ \\
\hline $\mathrm{V}_{1} \mathrm{As}_{2}$ & 39.82 g-k & $40.92 \mathrm{~g}-\mathrm{j}$ & $45.41 \mathrm{~h}-\mathrm{k}$ & $42.55 \mathrm{~h}-\mathrm{k}$ & $26.33 \mathrm{~h}-\mathrm{k}$ & 11.14 no \\
\hline $\mathrm{V}_{2} \mathrm{As}_{0}$ & $48.67 \mathrm{a}-\mathrm{c}$ & $50.78 \mathrm{a}-\mathrm{c}$ & $55.24 \mathrm{a}-\mathrm{d}$ & $52.58 \mathrm{a}-\mathrm{d}$ & $36.13 \mathrm{a}-\mathrm{d}$ & $20.87 \mathrm{~b}$ \\
\hline $\mathrm{V}_{2} \mathrm{As}_{1}$ & $47.44 \mathrm{a}-\mathrm{d}$ & $49.55 \mathrm{a}-\mathrm{d}$ & 54.01 a-e & 51.35 a-e & 34.90 a-e & $20.77 \mathrm{~b}$ \\
\hline $\mathrm{V}_{3} \mathrm{As}_{0}$ & 48.96 a-c & $51.07 \mathrm{a}-\mathrm{c}$ & $55.53 \mathrm{a}-\mathrm{d}$ & $52.87 \mathrm{a}-\mathrm{d}$ & 36.42 a-d & $16.43 \mathrm{~h}-\mathrm{k}$ \\
\hline $\mathrm{V}_{3} \mathrm{As}_{1}$ & $47.72 \mathrm{a}-\mathrm{d}$ & $49.83 \mathrm{a}-\mathrm{d}$ & 54.29 a-e & 51.63 a-e & 35.19 a-e & 15.99 i-1 \\
\hline $\mathrm{V}_{3} \mathrm{As}_{2}$ & 38.18 h-1 & 39.95 h-k & 44.44 i-1 & 41.57 i-k & $25.02 \mathrm{i}-1$ & $9.49 \mathrm{p}$ \\
\hline $\mathrm{V}_{4} \mathrm{As}_{0}$ & $37.58 \mathrm{i}-1$ & 39.69 h-k & $44.15 \mathrm{i}-1$ & $41.49 \mathrm{i}-1$ & $25.04 \mathrm{i}-1$ & $15.60 \mathrm{kl}$ \\
\hline $\mathrm{V}_{4} \mathrm{As}_{1}$ & $36.35 \mathrm{j}-\mathrm{m}$ & 38.46 i-1 & $42.92 \mathrm{j}-\mathrm{m}$ & $40.26 \mathrm{j}-\mathrm{n}$ & $23.81 \mathrm{j}-1$ & 15.171 \\
\hline $\mathrm{V}_{4} \mathrm{As}_{2}$ & $26.80 \mathrm{o}$ & $28.24 \mathrm{n}$ & $32.06 \mathrm{p}$ & $30.19 \mathrm{p}$ & $13.31 \mathrm{n}$ & $8.90 \mathrm{p}$ \\
\hline $\mathrm{V}_{5} \mathrm{As}_{1}$ & $45.63 \mathrm{~b}-\mathrm{f}$ & 47.74 b-e & $52.20 \mathrm{c}-\mathrm{f}$ & $49.54 \mathrm{c}-\mathrm{f}$ & $33.10 \mathrm{c}-\mathrm{f}$ & $21.65 \mathrm{ab}$ \\
\hline $\mathrm{V}_{5} \mathrm{As}_{2}$ & $36.09 \mathrm{j}-\mathrm{m}$ & $37.21 \mathrm{i}-\mathrm{m}$ & $41.68 \mathrm{k}-\mathrm{o}$ & $39.48 \mathrm{k}-\mathrm{n}$ & $22.59 \mathrm{k}-\mathrm{m}$ & $15.87 \mathrm{j}-1$ \\
\hline $\mathrm{V}_{6} \mathrm{As}_{0}$ & $45.81 \mathrm{~b}-\mathrm{f}$ & $47.92 \mathrm{~b}-\mathrm{e}$ & $52.38 \mathrm{c}-\mathrm{f}$ & $49.72 \mathrm{c}-\mathrm{f}$ & $33.27 \mathrm{~b}-\mathrm{f}$ & $21.16 \mathrm{ab}$ \\
\hline $\mathrm{V}_{6} \mathrm{As}_{1}$ & $44.57 \mathrm{c}-\mathrm{g}$ & $46.68 \mathrm{c}-\mathrm{f}$ & $51.14 \mathrm{~d}-\mathrm{g}$ & $48.48 \mathrm{~d}-\mathrm{g}$ & $32.04 \mathrm{~d}-\mathrm{g}$ & $20.71 b$ \\
\hline $\mathrm{V}_{6} \mathrm{As}_{2}$ & $34.70 \mathrm{k}-\mathrm{n}$ & $37.13 \mathrm{j}-\mathrm{m}$ & $41.62 \mathrm{k}-\mathrm{o}$ & 38.09 k-o & $21.53 \mathrm{k}-\mathrm{m}$ & 15.141 \\
\hline $\mathrm{V}_{7} \mathrm{As}_{0}$ & $44.46 \mathrm{c}-\mathrm{g}$ & $46.57 \mathrm{c}-\mathrm{f}$ & $51.03 \mathrm{~d}-\mathrm{g}$ & $48.37 \mathrm{~d}-\mathrm{g}$ & $31.92 \mathrm{~d}-\mathrm{g}$ & $18.23 \mathrm{c}-\mathrm{e}$ \\
\hline $\mathrm{V}_{7} \mathrm{As}_{1}$ & $43.22 \mathrm{~d}-\mathrm{h}$ & $45.33 \mathrm{~d}-\mathrm{g}$ & 49.79 e-h & $47.13 \mathrm{e}-\mathrm{h}$ & 30.69 e-h & $17.78 \mathrm{~d}-\mathrm{f}$ \\
\hline $\mathrm{V}_{7} \mathrm{As}_{2}$ & $33.351-n$ & $34.45 \mathrm{~lm}$ & 39.94 1-o & 36.74 1-o & $20.18 \mathrm{~lm}$ & 11.69 no \\
\hline $\mathrm{V}_{8} \mathrm{As}_{0}$ & $47.34 \mathrm{a}-\mathrm{d}$ & $49.45 \mathrm{a}-\mathrm{d}$ & 53.91 a-e & $51.25 \mathrm{a}-\mathrm{e}$ & 34.80 a-e & $18.14 \mathrm{c}-\mathrm{e}$ \\
\hline $\mathrm{V}_{8} \mathrm{As}_{1}$ & $46.10 \mathrm{~b}-\mathrm{f}$ & $48.21 \mathrm{~b}-\mathrm{e}$ & $52.67 \mathrm{~b}-\mathrm{f}$ & $50.01 \mathrm{~b}-\mathrm{f}$ & $33.57 \mathrm{~b}-\mathrm{f}$ & $17.72 \mathrm{~d}-\mathrm{g}$ \\
\hline $\mathrm{V}_{8} \mathrm{As}_{2}$ & $36.23 \mathrm{j}-\mathrm{m}$ & $38.33 \mathrm{i}-1$ & $43.15 \mathrm{j}-\mathrm{m}$ & $40.28 \mathrm{j}-\mathrm{n}$ & $23.06 \mathrm{j}-\mathrm{m}$ & $11.89 \mathrm{n}$ \\
\hline $\mathrm{V}_{9} \mathrm{As}_{0}$ & $52.01 \mathrm{a}$ & $54.12 \mathrm{a}$ & $58.58 \mathrm{a}$ & $55.92 \mathrm{a}$ & $39.48 \mathrm{a}$ & $19.09 \mathrm{c}$ \\
\hline $\mathrm{V}_{9} \mathrm{As}_{1}$ & $50.78 \mathrm{ab}$ & $52.89 \mathrm{ab}$ & $57.35 \mathrm{ab}$ & $54.69 \mathrm{ab}$ & $38.24 \mathrm{ab}$ & $18.61 \mathrm{~cd}$ \\
\hline $\mathrm{V}_{9} \mathrm{As}_{2}$ & $40.90 \mathrm{f}-\mathrm{j}$ & $42.34 \mathrm{f}-\mathrm{i}$ & $47.16 \mathrm{~g}-\mathrm{j}$ & $44.63 \mathrm{~g}-\mathrm{j}$ & $27.74 \mathrm{~g}-\mathrm{j}$ & $12.18 \mathrm{n}$ \\
\hline $\mathrm{V}_{10} \mathrm{As}_{1}$ & $43.13 \mathrm{~d}-\mathrm{h}$ & $45.24 \mathrm{~d}-\mathrm{g}$ & 49.70 e-h & $47.04 \mathrm{e}-\mathrm{h}$ & 30.60 e-h & $16.17 \mathrm{~h}-1$ \\
\hline $\mathrm{V}_{10} \mathrm{As}_{2}$ & $33.261-\mathrm{n}$ & $35.02 \mathrm{k}-\mathrm{m}$ & $39.18 \mathrm{~m}-\mathrm{o}$ & $36.65 \mathrm{~m}-\mathrm{o}$ & $20.43 \mathrm{~lm}$ & $9.62 \mathrm{p}$ \\
\hline $\mathrm{V}_{11} \mathrm{As}_{0}$ & $31.70 \mathrm{mn}$ & $33.81 \mathrm{~lm}$ & 38.27 no & 35.61 no & $22.43 \mathrm{k}-\mathrm{m}$ & $18.09 \mathrm{c}-\mathrm{e}$ \\
\hline
\end{tabular}




\begin{tabular}{lllllll}
\hline $\mathrm{V}_{11} \mathrm{As}_{1}$ & $30.46 \mathrm{no}$ & $32.57 \mathrm{mn}$ & $37.03 \mathrm{o}$ & $34.37 \mathrm{o}$ & $21.19 \mathrm{~lm}$ & $17.67 \mathrm{~d}-\mathrm{g}$ \\
$\mathrm{V}_{11} \mathrm{As}_{2}$ & $20.92 \mathrm{p}$ & $22.02 \mathrm{o}$ & $27.18 \mathrm{q}$ & $23.64 \mathrm{q}$ & $11.02 \mathrm{n}$ & $10.61 \mathrm{o}$ \\
$\mathrm{V}_{12} \mathrm{As}_{0}$ & $43.04 \mathrm{~d}-\mathrm{h}$ & $45.15 \mathrm{~d}-\mathrm{g}$ & $49.61 \mathrm{e}-\mathrm{h}$ & $46.95 \mathrm{e}-\mathrm{h}$ & $30.51 \mathrm{e}-\mathrm{h}$ & $19.01 \mathrm{c}$ \\
$\mathrm{V}_{12} \mathrm{As}_{1}$ & $41.81 \mathrm{e}-\mathrm{i}$ & $43.92 \mathrm{e}-\mathrm{h}$ & $48.38 \mathrm{f}-\mathrm{i}$ & $45.72 \mathrm{f}-\mathrm{i}$ & $29.27 \mathrm{f}-\mathrm{i}$ & $18.53 \mathrm{~cd}$ \\
$\mathrm{~V}_{12} \mathrm{As}_{2}$ & $31.93 \mathrm{mn}$ & $33.03 \mathrm{~m}$ & $38.52 \mathrm{~m}-\mathrm{o}$ & $35.66 \mathrm{no}$ & $18.77 \mathrm{~m}$ & $11.42 \mathrm{no}$ \\
$\mathrm{V}_{13} \mathrm{As}_{0}$ & $45.89 \mathrm{~b}-\mathrm{f}$ & $48.00 \mathrm{~b}-\mathrm{e}$ & $52.46 \mathrm{~b}-\mathrm{f}$ & $49.80 \mathrm{~b}-\mathrm{f}$ & $33.35 \mathrm{~b}-\mathrm{f}$ & $19.07 \mathrm{c}$ \\
$\mathrm{V}_{13} \mathrm{As}_{1}$ & $44.66 \mathrm{c}-\mathrm{g}$ & $46.77 \mathrm{c}-\mathrm{f}$ & $51.23 \mathrm{~d}-\mathrm{g}$ & $48.57 \mathrm{~d}-\mathrm{g}$ & $32.12 \mathrm{~d}-\mathrm{g}$ & $18.57 \mathrm{~cd}$ \\
$\mathrm{~V}_{13} \mathrm{As}_{2}$ & $34.78 \mathrm{k}-\mathrm{n}$ & $36.88 \mathrm{j}-\mathrm{m}$ & $41.70 \mathrm{k}-\mathrm{o}$ & $38.50 \mathrm{k}-\mathrm{o}$ & $21.62 \mathrm{k}-\mathrm{m}$ & $11.44 \mathrm{no}$ \\
$\mathrm{V}_{14} \mathrm{As}_{0}$ & $47.60 \mathrm{a}-\mathrm{d}$ & $49.71 \mathrm{a}-\mathrm{d}$ & $54.17 \mathrm{a}-\mathrm{e}$ & $51.51 \mathrm{a}-\mathrm{e}$ & $35.06 \mathrm{a}-\mathrm{e}$ & $17.56 \mathrm{~d}-\mathrm{g}$ \\
$\mathrm{V}_{14} \mathrm{As}_{1}$ & $46.37 \mathrm{~b}-\mathrm{e}$ & $48.48 \mathrm{~b}-\mathrm{e}$ & $52.94 \mathrm{~b}-\mathrm{f}$ & $50.28 \mathrm{~b}-\mathrm{f}$ & $33.83 \mathrm{~b}-\mathrm{f}$ & $17.10 \mathrm{e}-\mathrm{i}$ \\
$\mathrm{V}_{14} \mathrm{As}_{2}$ & $36.49 \mathrm{j}-\mathrm{m}$ & $37.59 \mathrm{i}-\mathrm{m}$ & $42.41 \mathrm{k}-\mathrm{n}$ & $39.88 \mathrm{j}-\mathrm{n}$ & $23.99 \mathrm{j}-1$ & $10.68 \mathrm{o}$ \\
$\mathrm{SE} \mathrm{value}$ & 1.56 & 1.55 & 1.45 & 1.45 & 1.46 & 0.3502 \\
$\mathrm{CV}(\%)$ & 6.56 & 6.23 & 5.26 & 5.59 & 8.82 & 3.77 \\
\hline
\end{tabular}

In a column means having similar letter (s) are statistically similar and those having dissimilar letter (s) differ significantly by DMRT at 0.05 level of probability

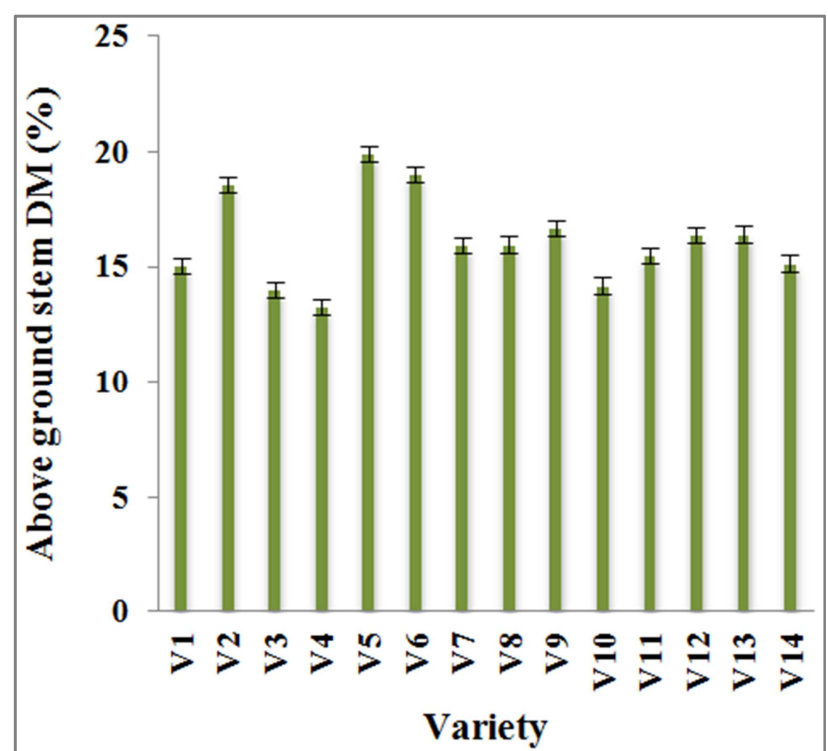

Figure 11. Effect of varieties on above ground stem dry matter content of potato (Vertical bar represents $S E$ value)

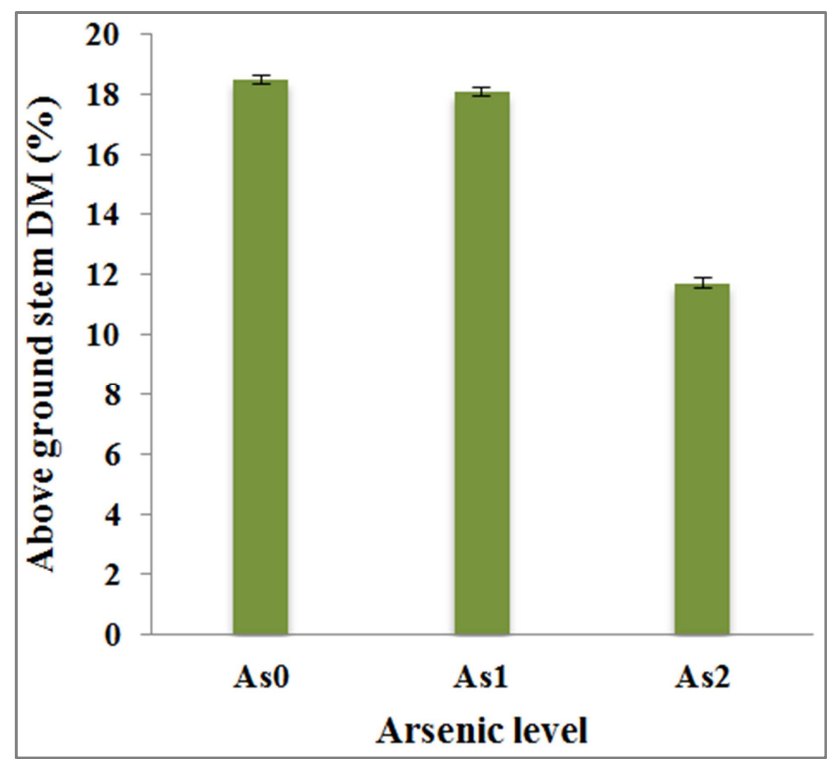

Figure 12. Effect of As levels on above ground stem dry matter content of potato (Vertical bar represents $S E$ value)

\subsection{Above Ground Stem Dry Matter Content}

Above ground stem dry matter content (\%) significantly influenced by the varieties and/or As levels (Figure 11, 12 and Table 7). 'Lady Rosetta' produced higher dry matter content of above ground stem (19.87 \%) whereas, the minimum (13.22 \%) was recorded from the variety 'Granola' (Figure 11). It is may be due to varietal characters. On the other hand, Figure 12 showed that, above ground stem dry matter content (\%) decreased with increasing As levels though $\mathrm{As}_{0}$ and $\mathrm{As}_{1}$ showed similar results. CarbonellBarrachina et al. [43] stated that root, stem and leaf dry biomass production of tomato and bean plants were increased with increasing As (III) levels in the nutrient solution. Present experiment showed that shoot dry matter content (\%) of

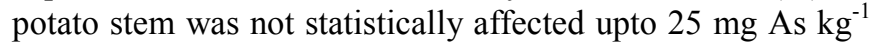
soil compared to control but at higher concentration $(50 \mathrm{mg}$ As $\mathrm{kg}^{-1}$ soil) treatment significantly decreased. In case of treatment combinations, the maximum $(22.10 \%)$ dry matter content of above ground stem was obtained from the combination of 'Lady Rosetta' with $\mathrm{As}_{0}$ which was statistically similar (21.65 and $21.16 \%$, respectively) with $\mathrm{V}_{5} \mathrm{As}_{1}$ and $\mathrm{V}_{6} \mathrm{As}_{0}$ whereas, the minimum (8.90\%) was recorded from the $\mathrm{V}_{4} \mathrm{As}_{2}$ which was statistically at par (9.49 and $9.62 \%$, respectively) with $\mathrm{V}_{3} \mathrm{As}_{2}$ and $\mathrm{V}_{10} \mathrm{As}_{2}$ (Table 7).

\section{Conclusion}

Considering the results of the present experiment, it may be concluded that growth of potato slowly decreased upto $25 \mathrm{mg}$ $\mathrm{As} \mathrm{kg}^{-1}$ soil and thereafter drastically decreased by increased As level. Among the potato varieties, 'Felsina' showed better performance irrespective of As levels. 'Felsina' combination

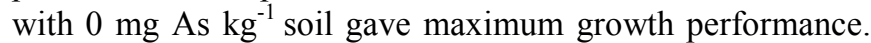

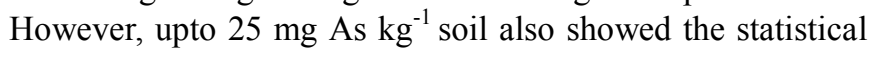
similar results in terms of growth parameters.

\section{Acknowledgements}

The authors acknowledge the funding of this study from the Ministry of Science and Technology-Government of the People's Republic of Bangladesh. 


\section{References}

[1] Stoeva, N. and Bineva, Tz. 2003. Oxidative changes and photosynthesis in oat plants grown in as-contaminated soil. Bulg. J. Plant Physiol. 29: 87-95.

[2] Kibria, G. 2013. Global Groundwater Arsenic Contamination Impacts on Agriculture Human Health Social Life. Sydney bashi bangla. Science \& Technology Article 36. 3 November 2013. p. 11 .

[3] Jiang, J. Q., Ashekuzzaman, S. M., Jiang, A., Sharifuzzaman, S. M. and Chowdhury, S. R. 2013. Arsenic Contaminated Groundwater and Its Treatment Options in Bangladesh. Intl. J. Environ. Res. Public Health. 10(1): 18-46.

[4] Chatterjee, D., Halder, D., Majumder, S., Biswas, A., Nath, B., Bhattacharya, P., Bhowmick, S., Mukherjee-Goswami, A., Saha, D., Hazra, R., Maity, P. B., Chatterjee, D., Mukherjee, A. and Bundschuh, J. 2010. Assessment of arsenic exposure from groundwater and rice in Bengal Delta Region, West Bengal, India. Water Res. 44: 5803-5812.

[5] Bhattacharya, P., Samal, A. C., Majumdar J. and Santra, S. C. 2009. Transfer of arsenic from groundwater and paddy soil to rice plant (Oryza sativa L.): A micro level study in West Bengal, India. World J. Agric. Sci. 5: 425-431.

[6] Bhattacharya, P., Samal, A. C., Majumdar, J. and Santra, S. C. 2010a. Accumulation of arsenic and its distribution in rice plant (Oryza sativa L.) in Gangetic West Bengal, India. Paddy Water Environ. 8(1): 63-70.

[7] Bhattacharya, P., Samal, A. C., Majumder, J. and Santra, S. C. 2010b. Arsenic contamination in rice, wheat, pulses and vegetables: A study in an Arsenic affected area of West Bengal, India. Water, Air Soil Pollut. 213(1): 3-13.

[8] Roberts, L. C., Hug, S. J., Dittmar, J., Voegelin, A., Kretzschmar, R., Wehrli, B., Cirpka, O. A., Saha, G. C. Ali, M. A. and Badruzzaman, A. B. M. 2010. Arsenic release from paddy soils during monsoon flooding. Nat. Geosci. 3: 53-59.

[9] Brammer, H. 2009. Mitigation of arsenic contamination in irrigated paddy soils in South and South-east Asia. Environ. Intl. 35(6): 856-863.

[10] Meharg, A. A., Williams, P. N., Adomako, E., Lawgali, Y. Y., Deacon, C., Villada, A., Sun, G., Zhu, Y. G., Feldmann, J., Raab, A., Zhao, F. J., Islam, R., Hossain, S. and Yanai, J. 2009. Geographical variation in total and inorganic arsenic content of polished (white) rice. Environ. Sci. Technol. 43(5): 1612-1617.

[11] Dahal, B. M., Fuerhacker, M., Mentler, A., Karki, K. B., Shrestha, R. R. and Blum, W. E. H. 2008. Arsenic contamination of soils and agricultural plants through irrigation water in Nepal. Environ. Pollut. 155(1): 157-163.

[12] Rahman, M. A., Hasegawa, H., Rahman, M. M., Rahman, M. A. and Miah, M. A. M. 2007. Accumulation of arsenic in tissues of rice plant (Oryza sativa L.) and its distribution in fractions of rice grain. Chemosphere. 69: 942-948.

[13] Huang, R. Q., Gao, S. F., Wang, W. L., Staunton, S. and Wang, G. 2006. Soil arsenic availability and the transfer of soil arsenic to crops in suburban areas in Fujian Province, southeast China. Sci. Total Environ. 368(2-3): 531-41.

[14] Norra, S., Berner, Z. A., Agarwala, P., Wagner, F., Chandrasekharam, D. and Stüben, D. 2005. Impact of irrigation with arsenic rich groundwater on soil and crops: a geochemical case study in West Bengal delta plain, India. Appli. Geochemis. 20: 1890-1906.

[15] Samal, A. C. 2005. An Investigation on Accumulation of Arsenic in Ecosystem of Gangetic West Bengal and Assessment of Potential Health Risk. Ph. D. thesis, University of Kalyani, India.

[16] Marin, A. R., Pezeshki, S. R., Masscheleyn, P. H. and Choi, H. S. 1993. Effect of dimethylarsinic acid (DMAA) on growth, tissue arsenic and photosynthesis of rice plants. J. Plant Nutr. 16: 865-880.

[17] Abedin, M. J. and Meharg, A. A. 2002a. Relative toxicity of arsenite and arsenate on germination and early seedling growth of rice (Oryza sativa L.). Plant Soil. 243: 57-66.

[18] Santra, S. C., Samal, A. C., Bhattacharya, P., Banerjee, S., Biswas, A. and Majumdar, J. 2013. Arsenic in Foodchain and Community Health Risk: A Study in Gangetic West Bengal. Procedia Environ. Sci. 18: 2-13.

[19] Abedin, M. J., Cresser, M. S., Meharg, A. A., Feldmann, J. and Cotter-Howells, J. 2002. Arsenic accumulation and metabolism in rice (Oryza sativa L.). Environ. Sci. Technol. 36(5): 962-968.

[20] Kabata-Pendias A., Pendias H. 1991. Trace elements in soils and plants, CRC Press, Boca Raton, FL.

[21] Liao, X. Y., Chen, T. B., Xie, H. and Liu, Y. R. 2005. Soil As contamination and its risk assessment in areas near the industrial districts of Chenzhou city, southern China. Environ. 791-798.

[22] FAOSTAT (FAO, Statistics Division). 2012. Statistical Database. Food and Agricultural Organization of the United Nations, Rome, Italy.

[23] Ali, M. A., Badruzzaman, A. B. M., Jalil, M. A., Hossain, M. D., Ahmed, M. F., Masud, A. A., Kamruzzaman, M. and Rahman, M. A. 2003. Fate of arsenic extracted with groundwater in fate of arsenic in the environment. Paper presented in the International Symposium on Fate of Arsenic in the Environment. Organized by BUET Bangladesh and UNU, Japan, February, 7.

[24] Gomez, K. A. and Gomez, A. A. 1984. Statistical procedure for agricultural research. Second Edn. Intl. Rice Res. Inst., John Wiley and Sons. New York. pp. 1-340.

[25] Talukdar, D. 2011. Effect of arsenic-induced toxicity on morphological traits of Trigonella foenum-graecum L. and Lathyrus sativus L. during germination and early seedling growth. Curr. Res. J. Biol. Sci. 3(2): 116-123.

[26] Rabbani, M. G. 1996. Performance of 13 potato varieties multiplied from microtubers. A report of Netherland Technical Assistant Unit, CDP, Khamarbari, Dhaka, pp. 27-47.

[27] Bashar, M. A. 1978. Testing six varieties of potato at Jaintiapur. Proc. 1st workshop Potato Res. Workers. PRC. BARI, Joydebpur, Dhaka, pp. 30-31.

[28] Jacobs, L. W., Keeny, D. R. and Walsh, L. M. 1970. Arsenic residue toxicity to vegetables crops grown on plain field sand. Agron. J. 62: 588-591. 
[29] Duel, L. E. and Swoboda, A. R. 1972. Arsenic toxicity to cotton and soybeans. J. Environ. Qual. 1: 317-320.

[30] Kabata-Pendias, A. and Pendias, H. 1992. Trace element in soil and plants. CRC, 2nd Edition. London, UK.

[31] Marin, A. R., Masscheleyn, P. H. and Patrick, J. W. H. 1992. The influence of chemical form and concentration of arsenic in rice growth and tissue arsenic concentration. Plant Soil. 139: $175-183$.

[32] Carbonell-Barrachina, A. A., Burlo, F. and Mataix, J. (1995). Arsenic uptake, distribution and accumulation in tomato plants: effect of arsenite on plant growth and yield. J. Plant Nutr. 18: 1237-1250.

[33] Abedin, M. J., Cottep-Howells, J. and Meharg, A. A. 2002b. Arsenic uptake and accumulation in rice (Oryza sativa L.) irrigated with contaminated water. Plant Soil. 240: 311-319.

[34] Jahan, L., Haque, S., Ullah, S. M. and Kibria, M. G. 2003. Effects of arsenic on some growth parameters of rice plant. Dhaka Univ. J. Biol. Sci. 12(1): 71-77.

[35] Juzl, M. and Stefl, M. 2002. The effect of leaf area index on potatoes yield in soils contaminated by some heavy metals. Rostlinna vyroba. 48(7): 298-306.

[36] Bavec, F. and Bavec, M. 2001. Chlorophyll meter readings of winter wheat cultivars and grain yield prediction. Commun. Soil Sci. Plant Anal. 32(17-18): 2709-2719.
[37] Güler, S., Macit, I., Koç, A. and Ibrikci, H. 2006. Estimating leaf nitrogen status of strawberry by using chlorophyll meter reading. J. Biol. Sci. 6(6): 1011-1016.

[38] Paivoke, A. 1983. The term effect of lead and arsenate on the growth and development, chlorophyll content and nitrogen fixation of the garden pea. Annals-Botanici-Fennici. 20: 297306.

[39] Merry, R. H., Tiller, K. G. and Altson, A. M. 1986. The effects of contamination of soil with copper, lead and arsenic on the growth and composition of plants, I. effects of season, genotype, soil temperature and fertilizers. Plant Soil. 91: 115128.

[40] Miteva, E. and Merakchiyska, M. 2002. Response of chloroplasts and photosynthetic mechanism of bean plants to excess arsenic in soil. Bulg. J. Agric. Sci. 8: 151-156.

[41] Hendry, G. A. F. and Price, A. H. 1993. Stress indicators: chlorophylls and carotenoids. In: Hendry, G. A. F., Grime, J. P. (Eds.), Methods in Comparative Plant Ecology. Chapman \& Hall, London, pp. 148-152.

[42] Kara, B. and Mujdeci, M. 2010. Influence of late-season nitrogen application on chlorophyll content and leaf area index in wheat. Sci. Res. Ess. 5(16): 2299-2303.

[43] Carbonell-Barrachina, A. A., Burlo, E., Burgos-Hernandez, A., Lopez, E. and Mataix, J. 1997. The influence of arsenic concentration on arsenic accumulation in tomato and bean plants. Sci. Hortic. 71: 167-176. 